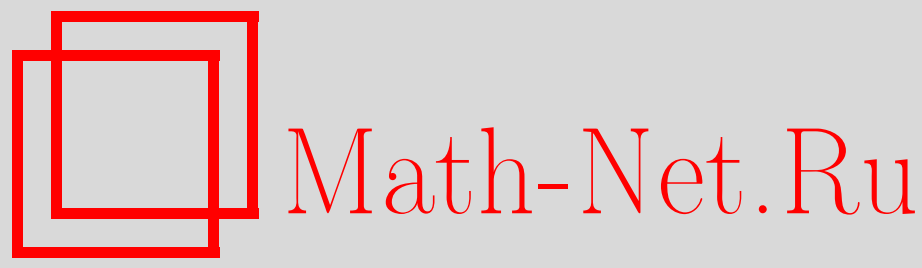

В. П. Маслов, Эффект измерительного прибора в "бозеконденсате" классического газа, в фазовом переходе и в экспериментах с отрицательным давлением, ТМФ, 2013, том 175, номер 1, 93-131

DOI: https://doi.org/10.4213/tmf8461

Использование Общероссийского математического портала Math-Net.Ru подразумевает, что вы прочитали и согласны с пользовательским соглашением http://www.mathnet.ru/rus/agreement

Параметры загрузки:

IP : 54.89 .56 .158

26 апреля 2023 г., 14:07:54

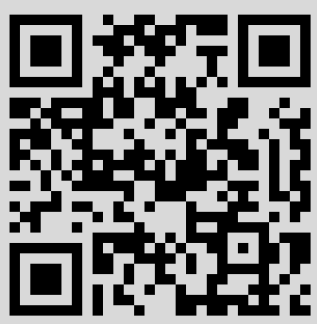




\title{
ЭФФЕКТ ИЗМЕРИТЕЛЬНОГО ПРИБОРА В "БОЗЕ-КОНДЕНСАТЕ" КЛАССИЧЕСКОГО ГАЗА, В ФАЗОВОМ ПЕРЕХОДЕ И В ЭКСПЕРИМЕНТАХ С ОТРИЦАТЕЛЬНЫМ ДАВЛЕНИЕМ
}

\begin{abstract}
Последовательно рассматривается новый подход к классической термодинамике при использовании асимптотических распределений теории чисел, обобщающих распределение Бозе-Эйнштейна. Обосновываются фазовый переход в жидкое состояние, термодинамика флюидов, а также поведение жидкости в области отрицательных давлений. Проводится сравнение с экспериментальными данными.
\end{abstract}

Ключевые слова: измерительный прибор, бозе-конденсат, фазовый переход первого рода, флюиды, отрицательное давление, влияние стенок сосуда, критерий Кнудсена, закон Клапейрона-Клаузиуса.

DOI: $10.4213 / \operatorname{tmf} 8461$

Памяти крупнейшего ученого и мудрейшего человека историка Юрия Александровича Полякова

\section{1. ВВЕДЕНИЕ}

Мое постоянное общение с экспериментаторами привело к некоторому абстрактному толкованию измерительного прибора и его роли в познании картины внешнего мира. В отличие от Маха, мы признаем объективное существование внешнего мира, но считаем совершенно необходимым тщательнейшим образом разобрать его отражение в образе, который "распознает" экспериментатор с помощью своего прибора. В квантовой теории есть термин "наблюдаемая величина". В классической термодинамике такой термин также необходимо было бы ввести.

Экспериментатор использует измерительные приборы, в том числе свои собственные глаза - они тоже являются своеобразным измерительным прибором. Известно выражение: "обман зрения". Точно так же грубый измерительный прибор может показывать нам картину, которую более точный измерительный прибор полностью

* Государственный исследовательский университет "Высшая школа экономики", Москва, Россия. E-mail: v.p.maslov@mail.ru 
изменит. Так, при близком рассмотрении картин художников-пуантилистов мы видим только отдельные точки, а на расстоянии (при грубом рассмотрении) возникают очертания предметов и их четкие границы.

Измерительный прибор, как фотоаппарат, должен быть согласован с масштабом того объекта, который исследует экспериментатор. Это соотношение между масштабом объекта и величиной геометрической разрешающей способности прибора является столь же важным, как наведение фотоаппарата на фотографируемый объект. Чем больше разрешающая способность, тем меньше диапазон, который данный прибор может подвергнуть исследованию.

Бор сформулировал свой принцип дополнительности, объяснив его вмешательством прибора в эксперимент. Мы говорим несколько о другом. В нашем случае прибор не вмешивается в эксперимент, но он в некоторой степени связан с наукой о распознавании образов.

Как распознает образ человек, описал знаменитый скульптор Роден: “Я беру камень и удаляю все лишнее". Очевидно, что каждый отдельный скульптор распознает свой отдельный образ.

Теория, которой придерживается данный экспериментатор, играет огромную роль, и поэтому математик должен "очистить" результаты эксперимента от индивидуального восприятия его экспериментатором. Известно, что в одно из крупнейших открытий прошлого века не поверил вначале сам экспериментатор (П. А. Черенков), хотя математически это явление уже содержалось в неявном виде.

Принцип, на котором основано распределение Больцмана, так же как и теория информации Шеннона и понятие энтропии Шеннона, базируется на том, что мы можем распознать все измеряемые объекты, и, следовательно, замена одного объекта на другой приводит к некоторому новому состоянию, к новой картине (фотографии).

Если мы не может отличить один предмет от другого, то замена предметов один на другой для нас не меняет фотографической картинки. Различие этих двух ситуаций такое же, как если бы мы меняли местами две карты открытой стороной или обратной стороной (рубашкой). Эта разница весьма существенная, поскольку если бы мы могли смотреть на рубашки карт через микроскоп, то могли бы различать достоинства карт не только по открытой стороне, но и по рубашкам, и тогда игра в карты была бы совершенно иной: можно было бы постепенно в процессе игры фиксировать в памяти, какая рубашка отвечает какой карте.

Таким образом, эта проблема связана, с одной стороны, с разрешающей способностью прибора и дискретностью времени, а с другой - с возможностями памяти, с запоминанием. Я хочу сказать, что если мы исследуем карточную игру с помощью методов старой теории вероятностей, то такая точность, которую нам обеспечивает микроскоп и компьютерная память, превращает эту игру в игру с открытыми картами. Однако разработка математической теории игры в покер остается столь же актуальной, как и до изобретения компьютеров.

Поэтому именно те приборы, которые были применены для исходных измерений, проверяющих аксиомы, должны проверять выполнение тех теорем, которые являются следствием построенной аксиоматики. Как логика доказательства не должна выходить за рамки той аксиоматики, которая была сформулирована, так и прибор 
не должен выходить за рамки того масштаба, который отвечает исследуемому объекту. Если мы будем применять другой прибор (плюс компьютерную память), то это равносильно тому, что мы учитываем в процессе доказательства какие-либо дополнительные условия, как это часто делают физики. Так, первый прибор может, например, четко показать границы объекта, а второй - зафиксировать, что точной границы объекта нет.

В термодинамике измеряется плотность молекул газа. Хотя молекулы газа отличаются друг от друга и подход Больцмана к исследованию молекул отвечает объективному миру, для определения плотности молекул отличие молекул друг от друга не играет роли. Если рассматривать плотность в маленьком фрагменте объема сосуда, содержащем порядка миллиона частиц, то оказывается, что плотность в этом фрагменте с точностью до тысячи частиц (до 0.1\%) совпадает со средней плотностью в сосуде и не зависит от нумерации частиц.

Таким образом, если мы в аксиоматику включили плотность как один из важнейших элементов термодинамики, то мы не можем ожидать, что в окончательных теоремах мы получим результат, который нам дает прибор распознавания, позволяющий различить частицы газа.

В классической системе уравнений Гамильтона для $N$ частиц, если даже гамильтониан не меняется при перестановке частиц, начальные условия таким свойством не обладают. Но начальные условия уравнения Лиувилля можно считать удовлетворяющими условиям симметрии.

Действительно, пусть

$$
\left\{q_{i}^{0}, p_{i}^{0}\right\}, \quad q_{i}^{0}=q_{i, 1}^{0}, q_{i, 2}^{0}, q_{i, 3}^{0}, \quad p_{i}^{0}=p_{i, 1}^{0}, p_{i, 2}^{0}, p_{i, 3}^{0},
$$

- начальные значения для системы Гамильтона, гамильтониан которых симметричен относительно перестановки $p_{i}, p_{j}$, а также перестановки $q_{i}, q_{j}$. Например,

$$
H(p, q)=\sum \frac{p_{i}^{2}}{2 m}+\sum \sum V\left(\left|q_{i}-q_{j}\right|\right) .
$$

Подставив начальные условия в гамильтониан (2), получим энергию

$$
E=H\left(p_{i}^{0}, q_{i}^{0}\right)
$$

Энергия сохраняется вдоль траекторий гамильтоновой системы.

Рассмотрим далее уравнение Лиувилля, отвечающее системе Гамильтона:

$$
\frac{\partial \rho}{\partial t}=\{H(p, q), \rho\}
$$

где $\{\cdot, \cdot\}$ - скобка Пуассона, с начальным условием $\rho(E), \rho(E) \in C^{\infty}$, где $E$ удовлетворяет (3). Это уравнение описывает распределение, отвечающее системе Гамильтона с заданными выше начальными условиями.

Уравнения Лиувилля и их начальные условия симметричны относительно перестановки как $p_{i}$ и $p_{j}$, так и $q_{i}$ и $q_{j}$. Эта симметрия сохраняется для решений. Согласно распределению Гиббса для гиббсовского ансамбля всякое распределение выражается через энергию. Следовательно, является симметричным по перестановке частиц. 
Впервые Шенберг произвел вторичное квантование этой системы в пространстве Фока [1], [2]. Это, разумеется, невозможно без предположения об идентичности частиц. Автор получил из вторично квантованной системы уравнение Власова при условии малости взаимодействия и большого числа частиц [2], [3]. Цепочки ББГКИ также симметричны относительно этих перестановок. Отсюда следует, что для любого распределения в задаче многих тел имеет место такая симметрия. Таким образом, мы приходим к перестановочности частиц, а значит, к распределению типа распределения Бозе-Эйнштейна для статистической физики классических частиц. Другие математически строгие аргументы, приводящие к распределению типа распределения Бозе-Эйнштейна, в виде лемм и теорем (см. [4]), а следовательно, и распределения классических частиц подчиняются законам теории чисел.

ЗАМЕчАНИЕ 1. Можно подходить к проблеме постоянной (с точностью до флуктуаций порядка $\sqrt{N}$ ) плотности по-другому, как мы это делаем в новой теории вероятностей [5]. Прибор различает частицы в данном фрагменте объема, содержащем около миллиона частиц. Прибор их отлично различает, но, наблюдая за ними через определенный промежуток времени, замечает, что, хотя, возможно, частицы в этом объеме все новые, их число с точностью до 1000 сохраняется. Поэтому мы условимся, что различие частиц для нас несущественно, и продолжаем измерять только их плотность. А следовательно, их число есть инвариант. Это обстоятельство формулируется как аксиома, и далее оказывается, что плотность при некотором значении одного из параметров в разных фрагментах объема будет различной, хотя частицы по-прежнему в каждом фрагменте, возможно, будут новыми.

Независимость плотности от перестановки частиц приводит к явлению, аналогичному явлению бозе-конденсата. Что может означать бозе-конденсат?

Прежде всего объясним более точно бозе-конденсат. Напомним вначале объяснение принципа тождественности частиц, которое приводится в учебнике [6]: «B классической механике одинаковые частицы (скажем, электроны), несмотря на тождественность их физических свойств, не теряют все же своей “индивидуальности". Именно, можно представить себе частицы, входящие в состав данной физической системы, в некоторый момент времени "перенумерованными" и в дальнейшем следить за движением каждой из них по своей траектории; тогда в любой момент времени частицы можно будет идентифицировать».

В квантовой механике, объясняют авторы, невозможно пронумеровать частицы и затем следить за поведением этих нумерованных частиц. Но и в термодинамике, как видно из предыдущего, прибор, измеряющий плотность, даже если и может пронумеровать частицы в некоторый момент времени в объеме, содержащем порядка миллиона молекул, он, тем не менее, не может в дальнейшем уследить за их номерами: в следующий момент времени ${ }^{1)}$ частицы в этом объеме будут другие и прибор должен будет их заново нумеровать. Поэтому в этом случае “тождественность" частиц чисто субъективная, но прибор и наши глаза их практически не различают. Поэтому с точки зрения нашего прибора мы рассматриваем только число частиц в объеме, а значит, должны рассматривать арифметическую симметрию сложения $a+b=b+a$.

1) Если в теории равновесной термодинамики меняется какой-либо параметр, то затем необходимо долго ждать, пока система снова придет в равновесное состояние. 
То обстоятельство, что плотность не зависит от перестановки частиц, приводит и к другому понятию энтропии, отличному от энтропии Больцмана, где каждая перестановка означает новое состояние. По определению энтропия Хартли есть логарифм от числа состояний. Эта новая энтропия вычислена в книге [7] в параграфе "Неравновесные ферми- и бозе-газы". Там речь идет о числе способов размещения $N$ одинаковых шаров по $G_{j}$ ящикам (см. также [5], [8]. Энтропия при этом имеет вид

$$
\begin{aligned}
& S=\sum_{j}\left\{\left(G_{j}+N_{j}\right) \ln \left(G_{j}+N_{j}\right)-N_{j} \ln N_{j}-G_{j} \ln G_{j}\right\}, \\
& S=\sum_{j} G_{j}\left[\left(1+\bar{n}_{j}\right) \ln \left(1+\bar{n}_{j}\right)-\bar{n}_{j} \ln \bar{n}_{j}\right] .
\end{aligned}
$$

Иначе говоря, энтропия имеет такой же вид, как в квантовом случае Бозе-Эйнштейна. Далее мы решаем задачу о максимуме энтропии при условиях

$$
\begin{gathered}
\sum_{j} N_{j}=\sum_{j} G_{j} \bar{n}_{j}=N, \\
\sum_{j} \varepsilon_{j} N_{j}=\sum_{j} \varepsilon_{j} G_{j} \bar{n}_{j}=M .
\end{gathered}
$$

В рассматриваемом случае $G_{j}=j^{\gamma}, \varepsilon_{j}=j$.

1.1. Радиус молекулы, длина свободного пробега и размерность бозераспределения. В обычной механике фазовое пространство для $N$ частиц имеет размерность $2 N$ : $N$ обобщенных координат и $N$ обобщенных импульсов. Число обобщенных координат совпадает, как правило, с числом степеней свободы. Те условия голономной связи, которые налагаются в механике, сокращают одновременно и число обобщенных координат, и число обобщенных импульсов.

Но в термодинамике одновременно используются два масштаба - микромасштаб и макромасштаб. Микромасштаб молекулы несоизмерим с объемом сосуда. Причем объем сосуда всегда трехмерный.

Рассмотрим примеры для определения размерности импульсного пространства. Имеет место точный математический факт: если газ достаточно разрежен (т. е. идеальный газ по Больцману-Максвеллу), то притяжения между молекулами нет. Предположим, что газ состоит из одноатомных молекул (аргон). Тогда число степеней свободы молекулы при $T=15^{\circ} \mathrm{C}$ и атмосферном давлении равно 3, теплоемкость равна $c_{v}=(3 / 2) R(R$ - газовая постоянная), что совпадает с экспериментом.

Для трехмерного бозе-газа, согласно [7] (§56 “Ферми- и бозе-газы элементарных частиц"), при адиабатическом процессе имеют место соотношения

$$
V T^{3 / 2}=\text { const }, \quad P V^{5 / 2}=\text { const }, \quad P T^{3 / 2}=\text { const. }
$$

Эти соотношения, как отмечается в [7], совпадают с адиабатой Пуассона для одноатомного газа.

При $\mu \rightarrow-\infty$ соотношение идеального трехмерного бозе-газа переходит в соотношение

$$
P V=V T=R T
$$

т. е. в соотношение идеального газа Больцмана-Максвелла, для которого $c_{p}-c_{v}=R$.

4 Теоретическая и математическая физика, т. 175, № 1, 2013 г. 
Отсюда следует, что

$$
\frac{c_{p}}{c_{v}}=\frac{5}{3}
$$

Пусть $T=T_{0}$, где $T_{0}$ - точка росы при $P_{0}=1$ атм. Нормируем $T / T_{0}=T_{\mathrm{r}}, P / P_{0}=P_{\mathrm{r}}$. При понижении температуры $T_{\mathrm{r}}<1, P_{\mathrm{r}}=T_{\mathrm{r}}^{3 / 2}$ образуется бозе-конденсат. Когда плотность бозе-конденсата будет настолько большой, чтобы расстояние между частицами стало достаточно малым для возникновения притяжения между ближайшими соседями, образуются шарообразные капли, которые будут виртуальными, пока их радиус не достигнет критического.

Теперь рассмотрим двухатомную молекулу (азот). Число степеней свободы этой молекулы равно 5. Рассмотрим пятимерный (по импульсам) газ Бозе-Эйнштейна (объем $V$ трехмерный!). После перехода при $\mu \rightarrow-\infty$ мы получим аналогично предыдущему закон $P V=R T$ и $c_{v}=(5 / 2) R$.

Повторяя рассуждения относительно аргона, при $T_{\mathrm{r}}<1$ получим $P_{\mathrm{r}}=T_{\mathrm{r}}^{7 / 2}$, пока плотность в бозе-конденсате не будет настолько большой, что возникнет притяжение и образуется поверхностное натяжение.

Аналогично для трехатомной молекулы (сероводород) получим число степеней свободы 6 , и процедура образования бозе-конденсата будет такой же для шестимерного бозе-газа (объем останется трехмерным): $P_{\mathrm{r}}=T_{\mathrm{r}}^{4}$. (Предполагается голономная связь между молекулами, т. е. расстояние между атомами не меняется.)

Таким образом, мы видим, что размерность импульсного пространства бозе-распределения совпадает с числом степеней свободы. Степени свободы у молекул, движущихся с разными скоростями, вообще говоря, различные. Поэтому среднее число степеней свободы, равное размерности импульсного пространства, для бозе-распределения может быть дробным.

В равновесной термодинамике есть еще понятие "время релаксации". Мы опустили температуру до точки росы и должны ждать, пока система уравновесится. Из вышесказанного, по-видимому, следует, что образование осязаемого кластера, в котором частицы в основном не покидают его границ, для сероводорода (трехатомная молекула) произойдет быстрее, чем для аргона.

Итак, если бы не было конденсации Бозе-Эйнштейна, точнее флуктуации, то откуда в идеальном газе Больцмана при понижении температуры взялось бы притяжение - квантовый эффект, имеющий место только между ближайшими соседями, в то время как в идеальном газе Больцмана "ближайшие соседи" находятся далеко друг от друга? Бозе-флуктуация есть математическое следствие независимости плотности от перестановки частиц. Только эта флуктуация может "приблизить соседей" настолько, что они начнут притягиваться. Чтобы объяснить "на пальцах" явление бозе-конденсата, рассмотрим пример.

ПримеР 1. Рассмотрим пример знаменитой теоремы теории чисел - решение старинной задачи, имеющей латинское название "partitio numerorum". В этой задаче участвует целое число $M$, которое разлагается на $N$ слагаемых, например $M=5$, $N=2$ :

$$
5=1+4=2+3,
$$

что дает 2 варианта решения этой задачи: $\mathcal{M}=2$. 
Если $M=10^{23}, N=1$, то вариант разложения всего один: $\mathcal{M}=1$. Если $M=10^{23}$ и $N=10^{23}$, то вариант разложения также только один - это сумма единиц, т. е. $\mathcal{M}=1$.

Очевидно, что найдется такое число $N_{\text {c }}$ при фиксированном $M$, при котором число вариантов разложения $\mathcal{M}$ будет максимально (это число, вообе говоря, не единственно). Энтропией Хартли называется величина $\log _{2} \mathcal{M}$. В точке, где она достигает своего максимума, возникает максимум энтропии.

Пусть задано разбиение $M=a_{1}+\cdots+a_{N}$ числа $M$ на $N$ слагаемых. Обозначим через $N_{j}$ количество слагаемых, в точности равных числу $j$, в правой части этого равенства. Тогда всего слагаемых будет $\sum_{j} N_{j}$, и это число равно $N$, поскольку мы знаем, что слагаемых всего $N$ штук. Далее, сумма слагаемых, равных $j$, равна $j N_{j}$, поскольку их $N_{j}$ штук, а сумма всех слагаемых тогда получается суммированием этих выражений по $j$, т. е. $\sum_{j} j N_{j}$, и она равна $M$. Именно,

$$
\sum_{i=1}^{\infty} N_{i}=N, \quad \sum_{i=1}^{\infty} i N_{i}=M .
$$

Неединственность указанного выше максимума и неопределенность числа этих максимумов позволили Эрдёшу получить результат лишь с точностью до $o(\sqrt{M})$.

Таким образом, имеет место теорема Эрдёша для системы двух диофантовых уравнений (5). Максимальное число решений этой системы достигается при выполнении соотношения

$$
N_{\mathrm{c}}=\beta^{-1} M_{\mathrm{c}}^{1 / 2} \ln M_{\mathrm{c}}+\alpha M_{\mathrm{c}}^{1 / 2}+o\left(M_{\mathrm{c}}^{1 / 2}\right), \quad \beta=\pi \sqrt{\frac{2}{3}},
$$

а коэффициент $\alpha$ определяется формулой $\beta / 2=e^{-\alpha \beta / 2}$.

Если в задаче (5) число $N$ увеличивать, а $M$ оставлять постоянным, то число решений будет убывать. Если суммы (5) отсчитывать от нуля, а не от единицы, т. е.

$$
\sum_{i=0}^{\infty} i N_{i}=M-N, \quad \sum_{i=0}^{\infty} N_{i}=N,
$$

то число решений не будет убывать, а будет оставаться постоянным.

Объясним этот факт. Задача Эрдёша-Ленера [9] заключается в разложении числа

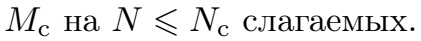

Разложение числа 5 на два слагаемых дает два варианта. Если мы включим также ноль, то получим три варианта: $5+0=3+2=4+1$. Таким образом, включение нуля дает возможность сказать, что мы разлагаем число на $k \leqslant n$ слагаемых. Действительно, разложение числа 5 на три слагаемых включает все предыдущие варианты: $5+0+0,3+2+0,4+1+0$ и добавляет новые варианты уже без нуля. Точка максимума при этом не сильно изменится [9], зато число вариантов не будет уменьшаться: нули позволяют максимуму оставаться постоянным, и энтропия никогда не будет убывать - выйдет после достижения максимума на константу. Это замечательное свойство энтропии и позволяет построить в общем случае неограниченную теорию вероятностей [10]. В физике этот эффект идентичен так называемому явлению бозе-конденсата. 
Спрашивается, чем отличаются арифметика и задача "partitio numerorum" от статистики Больцмана-Шеннона? Если мы полагаем, что $4+1$ и $1+4$ - это два разных варианта, то получаем статистику Больцмана-Шеннона. Число вариантов разложения $\mathcal{M}$ стремительно увеличивается. Таким образом, "некоммутативность" сложения дает дополнительно огромное число вариантов разложения и энтропия Хартли, равная логарифму числа вариантов, будет совпадать с энтропией Больцмана-Шеннона.

Итак, мы показали, что если добавить нуль и разложить число $M$ на $N$ слагаемых, то это равносильно решению уравнений (7), т. е. заданию соотношения для числа частиц и энергии. При этом число нулей в разложении будет сильно расти: если $M=5$, то при $N>3$ число нулей равно 22. Это и есть бозе-конденсат. Однако число единиц тоже велико, хотя и в два раза меньше, чем число нулей.

Рассматривать бозе-конденсат как число нулей очень наглядно и убедительно с точки зрения физиков, но неточно. Бозе-конденсат возникает не в точке, в которой энергия равна нулю, а в ее окрестности. Тем не менее, когда пишется соотношение для $\rho_{0} \delta(\mathbf{k})[11]$, где $\rho_{0}-$ плотность, а вектор $\mathbf{k}-$ импульс, для покоящегося бозе-конденсата, то эта запись верная, так как плотность есть предел:

$$
\rho_{0}=\lim _{N \rightarrow \infty, V \rightarrow \infty} \frac{N m}{V},
$$

где $N$ - число частиц, $V$ - объем, $m$ - масса частицы. Это значит, что при $N \rightarrow \infty$ колоколообразная функция вблизи нулевой энергии превращается в $\delta$-функцию.

Идеальной жидкостью будем называть жидкость без притяжения и без поверхностного натяжения. Это жидкость, которая при положительном давлении существует только совместно с насыщенным паром. Забегая вперед, отметим, что она может существовать также совместно с дырками.

При $\gamma>0$ идеальная жидкость - это результат обмана зрения: это тот же идеальный газ в конденсате с другой плотностью. Его можно описать, как в случае рассмотрения бозе-конденсата, в виде $\rho_{0} \delta(\mathbf{k})$, где $\rho_{0}-$ плотность конденсата. Она не может существовать без объема-ловушки подобно тому, как если в сосуде с газом есть отверстие, а вне сосуда - вакуум, то она, как бы вскипая, уйдет вместе с газом. Скорость частиц внутри этой жидкости в среднем такая же, как скорость частиц газа. Это соответствует тому, что температура в системе "жидкость-насыщенный пар" одна и та же в жидкости и в газе. Жидкость в закрытом сосуде - это застывшая на месте флуктуация $(\delta(\mathbf{k}))$, или, проще говоря, "покоящийся" бозе-конденсат (cp. [11]).

Кристаллики, которые возникают в пересыщенном растворе, отвечают бозе-конденсату только в том случае, если они не состоят из связанных между собой частиц и непрерывно обмениваются частицами с раствором, а кристаллики, как твердые тела, являются обманом зрения: мы просто не видим, что их частицы все время меняются с частицами раствора. То есть это вовсе не кристалл, а флуктуация, но флуктуация, которая сравнительно неподвижна.

Приведем пример такого явления. Предположим, что мы вдвоем одновременно подбрасываем две одинаковые монеты. Равновероятными являются события (орел, орел), (решка, решка), (орел, решка), (решка, орел). Допустим, что мы не можем 
узнать, где чья монета, после того, как монеты упали. Тогда число возможных вариантов 3: (орел, орел), (решка, решка) и (орел, решка). Если бросаний будет много, то вырастет кластер (орел, решка). Он будет состоять вперемешку из монет двоих бросающих. Это явление, очевидно, никого не удивляет. Точно так же неразличимость частиц прибором приведет к кластеру-флуктуации, которая называется бозе-конденсатом.

Чтобы составить представление о жидкости как о явлении, связанном с бозеконденсатом, проще всего представить себе такой вид жидкости, как стекло или аморфное тело. Именно оно больше всего отвечает изначальному эйнштейновскому трактованию бозе-конденсата как недвижущейся, неподвижной энергии, почти нулевой.

Итак, бозе-конденсат для классических частиц представляет собой некоторые специальные флуктуации плотности, и не более того. Мы можем сказать, что эта флуктуация содержит такое количество частиц, которое, во-первых, превышает критическое значение числа частиц при данной температуре и, во-вторых, можно говорить о плотности в этом "покоящемся флуктуационном кластере" в нашем сосуде с газом. А если мы говорим только о плотности в этом кластере, то это значит, что (как в примере с малым объемом в миллион частиц) нельзя говорить о числе частиц, которые в этом кластере как бы застыли и не двигаются. Это только видимость, через минуту все частицы или их часть могут быть, вообще говоря, заменены на другие, и нумерация частиц внутри “флуктуационного" кластера может изменяться в каждый момент времени. В следующий момент это может быть совершенно такая же картина, но с другими частицами.

Мы говорим о некотором фрагменте объема. На самом деле покоящиеся флуктуационные "кластеры" могут быть раскиданы по всему сосуду. Но если есть хотя бы маленькое земное притяжение, то покоящиеся флуктуации будут скапливаться на дне. Если рассмотреть сосуд с газом в виде шара (см. ниже), то в силу отталкивания, которое возникает на границе, такие флуктуации будут находиться вблизи центра шара. Повторяем еще раз, что ничего, кроме того факта, что там будет большая плотность частиц, обобщенная теория бозе-конденсата гарантировать не может.

Пусть возникает флуктуация типа покоящегося конденсата Бозе-Эйнштейна. При этом сами молекулы внутри этого флуктуационного фрагмента движутся, возможно, с теми же скоростями, что и молекулы газа, и если бы можно было их пронумеровать, то номера менялись бы очень быстро. Если называть эту флуктуацию вида конденсации Бозе-Эйнштейна идеальной или виртуальной жидкостью, то реальные молекулы жидкости движутся в ней с теми же скоростями, что и молекулы газа (насыщенного пара).

Чтобы яснее представить себе эту картину, вообразим полотно, накручивающееся с одного валика на другой. Между валиками под тканью из шланга дует сильный ветер. Мы видим тот "горб", который образуется между валиками, но не замечаем, что полотно движется.

Еще один пример, более физический: ветер гонит и хаотически раздувает металлическую пыль, а в центре помещен магнит. Мы видим только колоколообразную 
башенку из частиц пыли, которые сосредоточены с большой плотностью над магнитом, однако проскакивают этот "колокол" с большой скоростью.

Тем не менее при увеличении плотности бозе-конденсата наш прибор может зафиксировать границу изменения плотности и показать нам, что имеются более плотная фаза и менее плотная фаза. Значит, только исходный прибор может показать границу этой абстрактной жидкости, т. е. второй фазы.

Итак, при температуре, немного меньшей точки росы, возникают капельки жидкости. Они шарообразны даже в случае наличия земного притяжения (на которое, как правило, ссылаются физики, когда говорят, что граница "газ-жидкость" плоская). В капле и газе (насыщенном паре) давления разные из-за поверхностного натяжения. Следовательно, основное правило равновесия "пар-жидкость" - равенство давлений - выполняется, только если мы пренебрегаем поверхностным натяжением, а тем самым притяжением молекул жидкости, так как эти два эффекта неразрывно связаны. Наша концепция новой идеальной жидкости исходит именно из этого предположения (об отсутствии притяжения между молекулами).

Автор на своем (горьком) опыте почувствовал справедливость утверждения, высказанного Френкелем в работе [12]: “К однообразию и постоянству мы легко привыкаем, перестаем его замечать. Привычное кажется нам естественным, понятным, непривычное - неестественным и непонятным. . . . В сущности говоря, мы не можем понять, а можем только привыкнуть". Я не раскрывал полностью карты, пока не были объяснены все современные и новейшие экспериментальные данные термодинамики.

В результате можно сказать, что наш прибор и экспериментатор, который им пользуется, могут "распознать образ" жидкой фазы, а мы можем, исходя из постулата идеального газа, рассчитать число частиц жидкой фазы с определенной степенью точности (см. ниже), соответствующей “грубой модели” - термодинамике.

\section{2. НОВЫЙ ИДЕАЛЬНЫЙ ГАЗ}

В $D$-мерном случае энергия вырождения, т. е. при $\mu=0$, имеет вид

$$
E_{\mathrm{d}}=\int_{0}^{\infty} \frac{\left(|p|^{2} / 2 m\right) d \varepsilon}{e^{\left(|p|^{2} / 2 m\right) / T_{\mathrm{d}}}-1},
$$

где

$$
d \varepsilon=\text { const } \cdot \frac{|p|^{2}}{2 m} d p_{1} \ldots d p_{D}
$$

Отсюда мы получаем коэффициенты $C$ и $\lambda^{D}$ в формуле

$$
E_{\mathrm{d}}=C \lambda^{D} T_{\mathrm{d}}^{2+\gamma} \zeta\left(1+\frac{D}{2}\right) \Gamma\left(1+\frac{D}{2}\right) .
$$

В классическом случае значение $\mu=0$ отвечает не вырождению, а критическим значениям, которые помечаются индексом "c". Удобнее рассматривать другую нормировку

$$
d \varepsilon^{\prime}=\frac{1}{\Gamma(D / 2+1)} d \varepsilon
$$



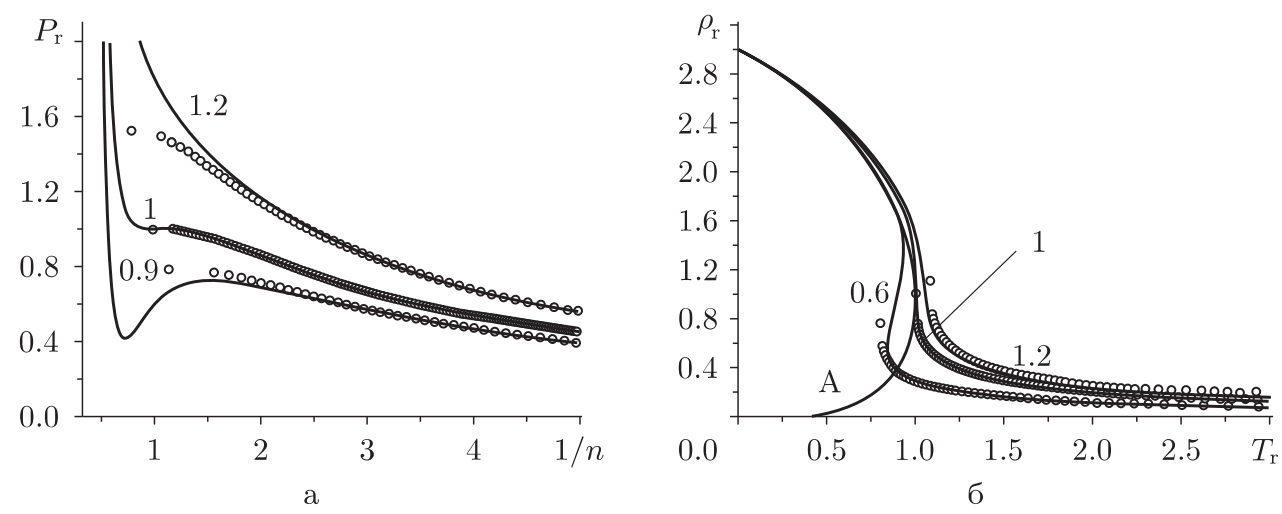

Рис. 1. Изотермы давления (а) и изобары плотности (б) для уравнения Ван-дер-Ваальса показаны сплошными линиями. Линии, образованные кружками, построены по вычислениям при $\gamma=0.312$ для $\varphi(V)=V$ (т. е. идеальный бозе-газ), $Z_{\mathrm{c}}=3 / 8, P_{\mathrm{r}}=P / P_{\mathrm{c}}, n=N / N_{\mathrm{c}}$. Линия А - бинодаль. Числа у кривых - значения $T_{\mathrm{r}}(\mathrm{a})$ и значения $P_{\mathrm{r}}$ (б).

для энергии и, соответственно,

$$
d \varepsilon^{\prime \prime}=\frac{1}{\Gamma(D / 2)} d \varepsilon
$$

для числа частиц $N$.

Переход к координатам Ван-дер-Ваальса приводит к коэффициенту $C \lambda^{D}$ вида $\tilde{\lambda}^{\gamma-\gamma_{c}}$, где $\gamma_{c}$ определяется из экспериментальных значений величины $\left.Z\right|_{\mu=0}=$ $\zeta\left(\gamma_{\mathrm{c}}+2\right) / \zeta\left(\gamma_{\mathrm{c}}+1\right), \zeta(x)$ - дзета-функция Римана. Ниже мы будем опускать волну у коэффициента $\lambda$.

После этого удобнее перейти к полилогарифму $\mathrm{Li}_{2+\gamma}\left(e^{-\mu / T_{\mathrm{r}}}\right)$ для энергии и полилогарифму $\operatorname{Li}_{1+\gamma}\left(e^{-\mu / T_{\mathrm{r}}}\right)$ для числа частиц:

$$
E=T_{\mathrm{r}}^{2+\gamma} \operatorname{Li}_{2+\gamma}(a), \quad N=T_{\mathrm{r}}^{1+\gamma} \operatorname{Li}_{1+\gamma}(a),
$$

где $a$ - активность $\left(a=e^{-\mu / T_{\mathrm{r}}}\right)$. Функция $\operatorname{Li}_{s}(x)$, введенная в $(14)$, называется полилогарифмом и определяется как

$$
\operatorname{Li}_{s}(x)=\frac{1}{\Gamma} \int_{0}^{\infty} \frac{t^{s-1} d t}{\left(e^{t} / x\right)-1} .
$$

Тогда фактор сжимаемости равен

$$
Z=\frac{E}{N T}=\frac{\operatorname{Li}_{2+\gamma}(a)}{\operatorname{Li}_{1+\gamma}(a)}, \quad Z_{\mathrm{c}}=\frac{\zeta(\gamma+2)}{\zeta(\gamma+1)}, \quad D=2 \gamma+2
$$

Коэффициент $\lambda$ здесь сокращается. Параметр $\gamma$ мы выбираем для каждого газа из экспериментальных значений для критического фактора сжимаемости. Ниже показано, что распределение Бозе-Эйнштейна для размерности $2 \gamma+2$ будет совпадать с экспериментальными данными и для всей критической изотермы. 


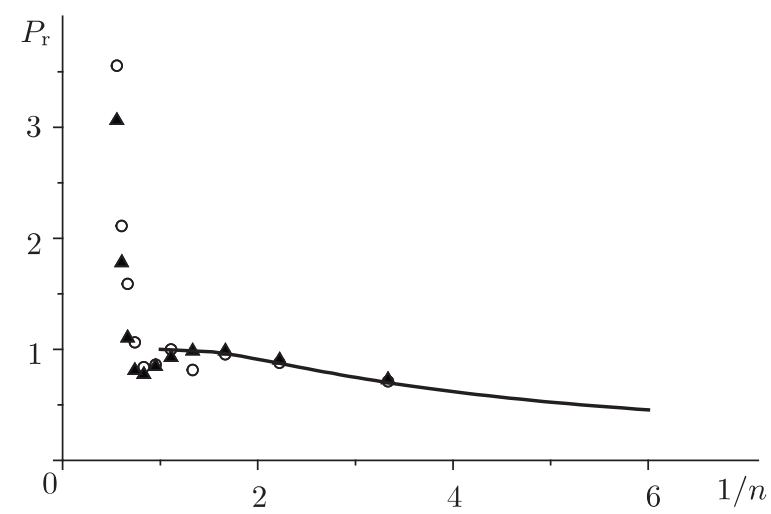

Рис. 2. Критические изотермы для системы Леннарда-Джонса. Кружки и треугольники соответствуют численным вычислениям, а кривая - идеальному бозе-газу при $\gamma=0.24$.

Связь известных экспериментальных данных с приведенной выше концепцией теории чисел продемонстрировали Апфельбаум и Воробьев [13] (на рис. 1-5 приведены графики из этой работы).

В нашей задаче мы рассматриваем трехмерный газ и жидкость. Поэтому объем $V$ всегда трехмерный. Дробная размерность по импульсам отвечает дробному числу степеней свободы. Нужно учитывать, что независимость плотности и, следовательно, всей исходной постановки задачи от перестановки частиц является существенным ограничением числа степеней свободы: у частиц с разными скоростями оно, вообще говоря, разное. Число степеней свободы $D$, которому отвечает важный параметр $\gamma(D=2 \gamma+2)$, - это усредненное число по всем молекулам, аналогично тому, как температура есть усредненная энергия.

\section{3. ОТРИЦАТЕЛЬНЫЕ ЗНАЧЕНИЯ ПАРАМЕТРА $\gamma$ И ФАЗОВЫЙ ПЕРЕХОД В ИДЕАЛЬНУЮ ЖИДКОСТЬ}

Газовая спинодаль, по-новому определенная как геометрическое место точек изотерм нового идеального газа, при максимуме энтропии образуется в точках, где химический потенциал $\mu$ равен нулю. Следовательно, на диаграмме $\left(Z, P_{\mathrm{r}}\right)$ она яв-

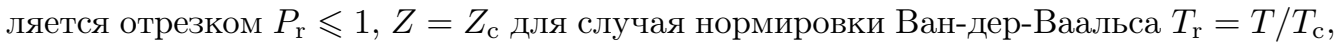
$P_{\mathrm{r}}=P / P_{\mathrm{c}}$. При $T_{\mathrm{r}} \leqslant 1$ возникает бозе-конденсат и, следовательно, для жидкой фазы на спинодали величина $N=T_{\mathrm{r}}^{\gamma_{c}+1} \zeta\left(\gamma_{\mathrm{c}}+1\right)$ на жидкой изотерме остается постоянной. Это значит, что изотерма жидкой фазы, отвечающая температуре $T$, имеет вид

$$
Z=\frac{P_{\mathrm{r}}}{T_{\mathrm{r}} N}=\frac{P_{\mathrm{r}}}{T_{\mathrm{r}}^{\gamma_{\mathrm{c}}+2} \zeta\left(\gamma_{\mathrm{c}}+1\right)}
$$

Все изотермы жидкой фазы (в том числе и критическая изотерма при $T_{\mathrm{r}}=1$ ) проходят через начало координат $Z=0, P_{\mathrm{r}}=0$ и далее попадают в отрицательную 


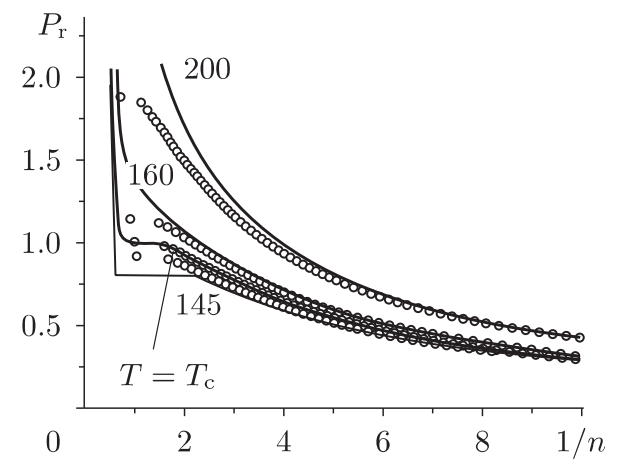

$\mathrm{a}$

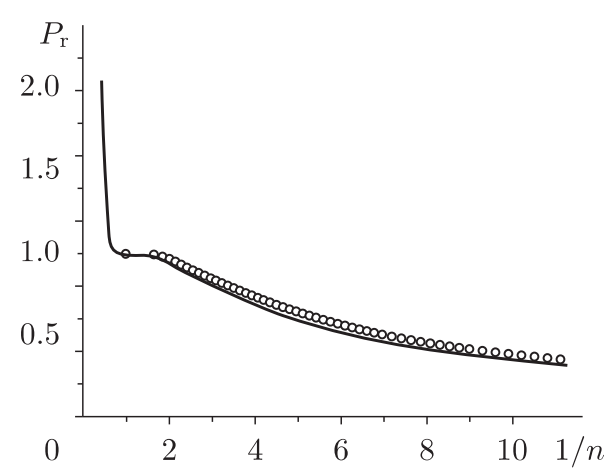

б

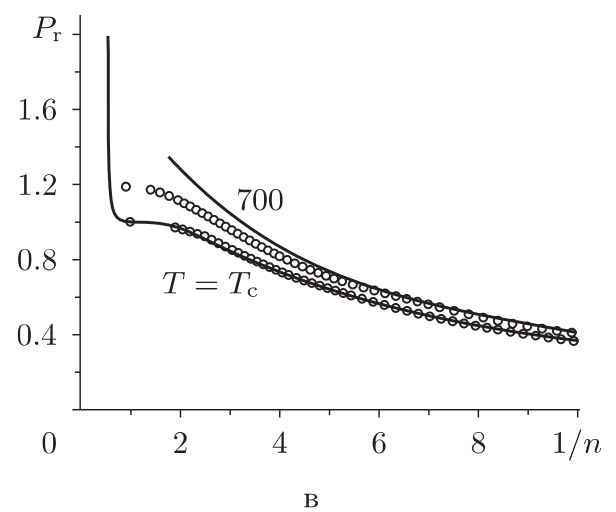

Рис. 3. Изотермы для аргона (а), воды (б) и меди (в). Сплошные линии соответствуют экспериментальным данным. Линия, образованная кружками, построена в соответствии с изотермой идеального бозе-газа. Величина $Z_{\mathrm{c}}=$ $\zeta(\gamma+2) / \zeta(\gamma+1)$ принимает значения $0.29(\mathrm{a}), 0.23$ (б), $0.39\left(\right.$ в), $P_{\mathrm{r}}=P / P_{\mathrm{c}}$, $n=N / N_{\text {c }}$. Числа у кривых - значения температуры в Кельвинах.

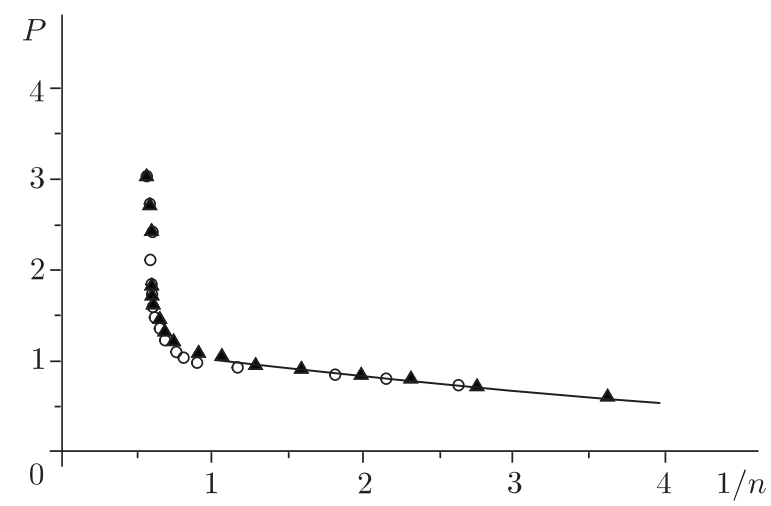

Рис. 4. Изотермы для воды. Кружки и треугольники соответствуют экспериментальным данным, а кривая - вычислениям для бозе-газа. 


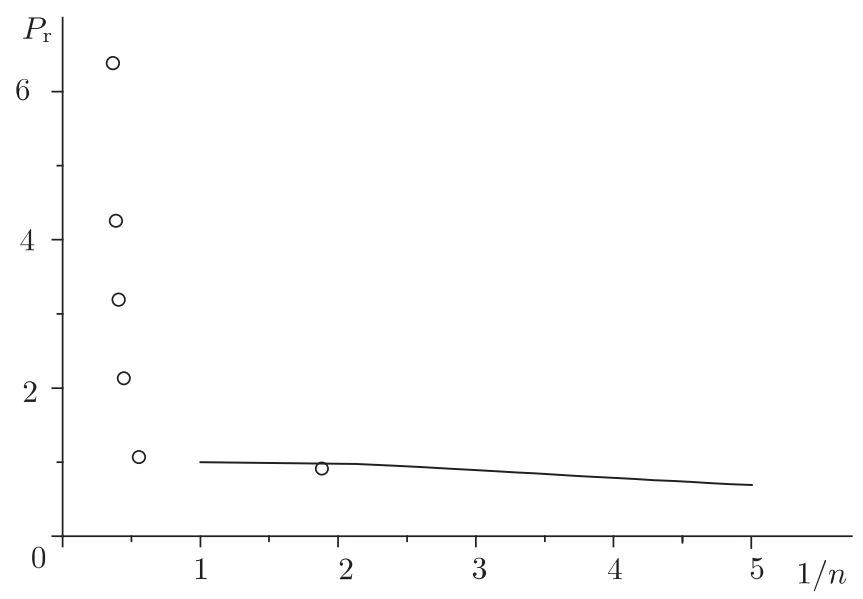

Рис. 5. Критические изотермы для ртути. Кружки соответствуют экспериментальным данным, а кривая - вычислениям для бозе-газа.

область (или на второй лист). Точка $Z=0$ отвечает параметру $\gamma=0$ и, следовательно, продолжению для $\gamma<0$, поскольку при $\mu=0$ давление

$$
P_{\mathrm{r}}=T_{\mathrm{r}}^{2+\gamma} \frac{\zeta(2+\gamma)}{\zeta\left(2+\gamma_{\mathrm{c}}\right)}
$$

продолжается для $0>\gamma>-1$.

Мы увидим ниже, что величина $Z$ при $\mu / T \rightarrow o(1 / \ln N)$ также положительна, и, следовательно, спинодаль при $0>\gamma>-1$ дает второй лист на диаграмме $(Z, P)$, который удобнее отобразить на отрицательный квадрант.

В предположении, что при $T_{\mathrm{r}}=1$ переход в жидкую фазу не осуществляется, мы приравниваем химические потенциалы $\mu$ и $\tilde{\mu}$ для "жидкой" и “газовой” фаз на изотерме $T_{\mathrm{r}}=1$ (этот факт доказывается ниже). Затем мы находим точку $\mu-$ точку перехода в "жидкую" фазу при $T_{\mathrm{r}}<1$, приравнивая химические потенциалы "жидкой" и "газовой" фаз.

В настоящем разделе мы найдем точку изотермы-изобары жидкости при медлен-

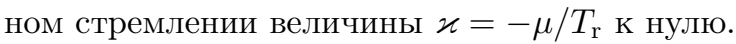

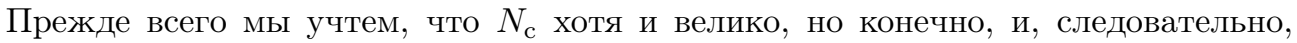
нужно использовать парастатистическую поправку. Напомним соотношение для $\Omega$-потенциала:

$$
\Omega=-\Lambda^{\gamma-\gamma_{\mathrm{c}}} T_{\mathrm{r}} \sum_{i} \ln \left(G_{i} \frac{1-e^{\left(\mu-\varepsilon_{i}\right) / T_{\mathrm{r}}} N}{1-e^{\left(\mu-\varepsilon_{i}\right) / T_{\mathrm{r}}}}\right) .
$$

где $\varepsilon_{i}=i, G_{i}=i^{\gamma+1}$. Из (4) получаем

$$
N=\sum_{i}\left(\frac{i^{\gamma}}{e^{b i+\varkappa}-1}-\frac{N i^{\gamma}}{e^{b N i+\varkappa}}\right) .
$$


Применяя формулу Эйлера-Маклорена с учетом параметра $\gamma$, получаем

$$
\sum_{i=1}^{n}\left(\frac{i^{\gamma}}{e^{b i+\varkappa}-1}-\frac{k i^{\gamma}}{e^{b k i+\varkappa}}\right)=\frac{1}{\alpha} \int_{0}^{\infty}\left(\frac{1}{e^{b x+\varkappa}-1}-\frac{k}{e^{b k x+\varkappa}-1}\right) d x^{\alpha}+R
$$

где $\alpha=\gamma+1, k=N$ и $b=1 / T_{\mathrm{r}}$. Здесь остаток $R$ удовлетворяет оценке

$$
|R| \leqslant \frac{1}{\alpha} \int_{0}^{\infty}\left|f^{\prime}(x)\right| d x^{\alpha}, \quad f(x)=\frac{1}{e^{b x+\varkappa}-1}-\frac{k}{e^{k(b x+\varkappa)}-1} .
$$

Вычисляя производную, получаем

$$
\begin{gathered}
f^{\prime}(x)=\frac{b k^{2} e^{k(b x+\varkappa)}}{\left(e^{k(b x+\varkappa)}-1\right)^{2}}-\frac{b e^{b x+\varkappa}}{\left(e^{b x+\varkappa}-1\right)^{2}} \\
|R| \leqslant \frac{b^{-\alpha}}{\alpha} \int_{0}^{\infty}\left|\frac{k^{2} e^{k(y+\varkappa)}}{\left(e^{k(y+\varkappa)}-1\right)^{2}}-\frac{e^{y+\varkappa}}{\left(e^{y+\varkappa}-1\right)^{2}}\right| d y^{\alpha} .
\end{gathered}
$$

Имеем также

$$
\frac{e^{y}}{\left(e^{y}-1\right)^{2}}=\frac{1}{y^{2}}+\psi(y),
$$

где $\psi(y)$ гладкая и $|\psi(y)| \leqslant C(1+|y|)^{-2}$. Подставляя эту формулу в $(21)$, получаем

$$
\begin{aligned}
|R| & \leqslant \frac{b^{-\alpha}}{\alpha} \int_{0}^{\infty}|\psi(k(y+\varkappa))-\psi(y+\varkappa)| d y^{\alpha} \leqslant \\
& \leqslant k^{-\alpha} b^{-\alpha} \int_{k \varkappa}^{\infty}|\psi(y)| d y^{\alpha}+b^{-\alpha} \int_{\varkappa}^{\infty}|\psi(y)| d y \leqslant C b^{-\alpha}
\end{aligned}
$$

с некоторой постоянной $C$. Например, если $\varkappa \sim(\ln k)^{-1 / 4}$, то $|R|$ имеет оценку

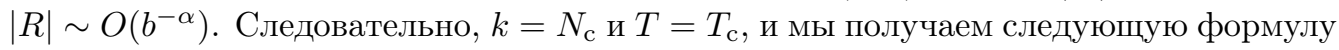
для интеграла при $\mu=0$ :

$$
M=\frac{\lambda^{\gamma-\gamma_{c}}}{\alpha \Gamma(\gamma+2)} \int \frac{\xi d \xi^{\alpha}}{e^{b \xi}-1}=\frac{\lambda^{\gamma-\gamma_{c}}}{b^{1+\alpha}} \int_{0}^{\infty} \frac{\eta d \eta^{\alpha}}{e^{\eta}-1},
$$

где $\alpha=\gamma+1$. Следовательно,

$$
b=\frac{1}{M^{1 /(1+\alpha)}}\left(\frac{\lambda^{\gamma-\gamma_{c}}}{\alpha \Gamma(\gamma+2)} \int_{0}^{\infty} \frac{\xi d \xi^{\alpha}}{e^{\xi}-1}\right)^{1 /(1+\alpha)} .
$$

Получаем (см. [14])

$$
\begin{aligned}
N \lambda^{\gamma-\gamma_{c}}= & \int_{0}^{\infty}\left(\frac{1}{e^{b \xi}-1}-\frac{k}{e^{k b \xi}-1}\right) d \xi^{\alpha}+O\left(b^{-\alpha}\right)= \\
= & \frac{1}{b^{\alpha}} \int_{0}^{\infty}\left(\frac{1}{e^{\xi}-1}-\frac{1}{\xi}\right) d \xi^{\alpha}+\frac{1}{b^{\alpha}} \int_{0}^{\infty}\left(\frac{1}{\xi}-\frac{1}{\xi(1+(k / 2) \xi)}\right) d \xi^{\alpha}- \\
& -\frac{k^{1-\alpha}}{b^{\alpha}} \int_{0}^{\infty}\left(\frac{k^{\alpha}}{e^{k \xi}-1}-\frac{k^{\alpha}}{k \xi(1+(k / 2) \xi)}\right) d \xi^{\alpha}+O\left(b^{-\alpha}\right)= \\
= & \frac{c(\gamma)}{b^{\alpha}}\left(k^{1-\alpha}-1\right)+O\left(b^{-\alpha}\right) .
\end{aligned}
$$


Положив $k=\left.N\right|_{\tilde{\mu} / T_{\mathrm{r}}=o(1)}(N \gg 1)$, имеем

$$
\left.N\right|_{\tilde{\mu} / T_{\mathrm{r}}=o(1)} \approx\left(\lambda^{\gamma-\gamma_{c}} c(\gamma)\right)^{1 /(1+\gamma)} T_{\mathrm{r}}, \quad c(\gamma)=\int_{0}^{\infty}\left(\frac{1}{\xi}-\frac{1}{e^{\xi}-1}\right) \xi^{\gamma} d \xi
$$

Соотношение $N=T_{\mathrm{r}}^{\gamma_{\mathrm{c}}+1} \zeta\left(\gamma_{\mathrm{c}}+1\right)$ сопрягается с линейным соотношением $N=$ $A(\gamma) T_{\mathrm{r}}$, где $A(\gamma)=\left(\lambda^{\gamma-\gamma_{\mathrm{c}}} c(\gamma)\right)^{1 /(1+\gamma)}$, при $P<0$.

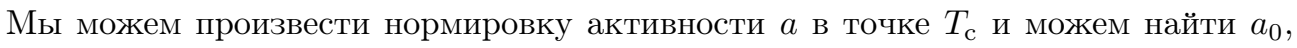

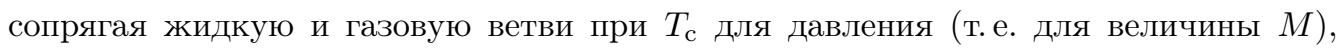
чтобы фазового перехода на критической изотерме при $T_{\mathrm{r}}=1$ не было.

Далее будем нормировать активности при $T_{\mathrm{r}}<1$ на вычисленное ниже значение $a_{0}$. Тогда химические потенциалы (в термодинамике - термодинамические потенциалы Гиббса для жидкой и газовой ветвей) будут совпадать и, следовательно, при $T_{\mathrm{r}}=1$ фазового перехода "газ-жидкость" не будет.

Теперь, чтобы изохора-изотерма "несжимаемой жидкости" имела место, мы должны построить ее, учитывая $N_{\mathrm{c}}=\zeta\left(\gamma_{\mathrm{c}}+1\right)$, т. е. для

$$
N\left(T_{\mathrm{r}}\right)=T_{\mathrm{r}}^{\gamma_{\mathrm{c}}+1} \zeta\left(\gamma_{\mathrm{c}}+1\right)
$$

Мы получаем значение $\gamma\left(T_{\mathrm{r}}\right)$ из неявного уравнения

$$
A(\gamma)=T_{\mathrm{r}}^{\gamma_{\mathrm{c}}} \zeta\left(\gamma_{\mathrm{c}}+1\right)
$$

Тем самым для каждого $T_{\mathrm{r}}<1$ мы найдем кривую спинодали (т. е. точки, где $\left.\tilde{\mu} \sim T_{\mathrm{r}}\left(\ln N\left(T_{\mathrm{r}}\right)\right)^{-1 / 4}\right)$ в области отрицательных $\gamma$ :

$$
\lambda^{-\left(\gamma-\gamma_{\mathrm{c}}\right) /(1+\gamma)}(c(\gamma))^{1 /(1+\gamma)}=T_{\mathrm{r}}^{\gamma_{\mathrm{c}}} \zeta\left(\gamma_{\mathrm{c}}+1\right)
$$

Из двух значений $\gamma$ решения (23) мы выбираем наименьшее (наибольшее по модулю) и обозначаем его $\gamma\left(T_{\mathrm{r}}\right)$. В частности, при $T_{\mathrm{r}}=1$ обозначаем $\gamma_{0}=\gamma(1)$.

Второе решение имеет место для некоторых металлов (ртуть, цезий и др.) и отвечает метастабильному состоянию. Реальное взаимодействие между частицами дает существенно большее отклонение второго решения от $\gamma_{0}$ (см. [15]).

Пусть $a_{\mathrm{g}}=e^{-\mu / T_{\mathrm{r}}}-$ активность газа, $a_{\mathrm{l}}=e^{-\tilde{\mu} / T_{\mathrm{r}}}-$ активность жидкости. Приведем условие равенства $M$ и активностей в точке фазового перехода (см. раздел 6):

$$
\begin{gathered}
T_{\mathrm{r}}^{\gamma_{\mathrm{c}}} \operatorname{Li}_{2+\gamma_{\mathrm{c}}}\left(a_{\mathrm{g}}\right)=\lambda^{-\left|\gamma\left(T_{\mathrm{r}}\right)\right|-\gamma_{\mathrm{c}}} T_{\mathrm{r}}^{-\left|\gamma\left(T_{\mathrm{r}}\right)\right|} \operatorname{Li}_{2-\left|\gamma\left(T_{\mathrm{r}}\right)\right|}\left(\frac{a_{\mathrm{l}}}{a_{0}}\right), \\
\frac{\lambda^{\gamma_{0}-\gamma_{\mathrm{c}}}}{\zeta\left(2+\gamma_{0}\right)} \operatorname{Li}_{2+\gamma_{0}}\left(a_{0}\right)=1, \quad a_{\mathrm{g}}=\frac{a_{\mathrm{l}}}{a_{0}} .
\end{gathered}
$$

ОПРЕДЕЛЕНИЕ 1. Равенство $a_{\mathrm{g}}=a_{\mathrm{l}} / a_{0}$ будем называть нормировкой активности на критической изотерме.

Равенства (24) и (25) определяют значение химического потенциала $\mu=\tilde{\mu}=$ $T_{\mathrm{r}} \ln a_{\mathrm{g}}$, при котором происходит фазовый переход “газа" в “жидкость".

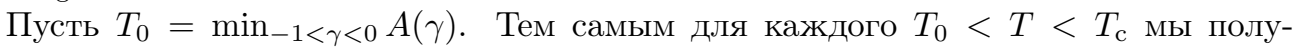
чим значение редуцированной активности жидкости $a_{\mathrm{r}}=a_{\mathrm{l}} / a_{0}$, что соответствует нормировке Ван-дер-Ваальса. 
ЗАмечАниЕ 2. В термодинамике критические значения $T_{\mathrm{c}}, P_{\mathrm{c}}, \rho_{\mathrm{c}}$ получены экспериментально почти для всех газов, поэтому критическое число степеней свободы может быть заранее задано. Параметр $\lambda\left(1.6<\lambda<3, T_{\mathrm{r}}>1 / 3\right)$ определяется из совпадения бинодали в тройной точке, полученной из эксперимента.

\section{4. ДЫРКИ В БОЗЕ-КОНДЕНСАТЕ}

Молекулы идеального газа можно представить себе как крохотные шарики. Представим себе дырки, экситоны в стекле, такие же, как шарики, но пустые (без вещества) молекулы. Очевидно, что если перемешать в стекле такие шарики хаотическим образом, то хаос в стекле еще увеличится. А это значит, что при наличии дырок энтропия увеличится. Поэтому, чтобы достичь максимума энтропии, надо еще "намешать" туда дырок.

В нашей концепции дырки возникают при $\gamma<0$.

В модели идеальной жидкости мы игнорируем притяжение, а это значит, что при "растяжении" жидкости, приводящем к возникновению дырок, жидкость не сопротивляется (как песок: на сжатие он несжимаем, а на "растяжение" не сопротивляется; ср. приложение в книге [16]).

Раз нет притяжения, то нет и отрицательного давления при "растяжении", т. е. образования дырок. Если $\gamma<0$, то плоскость $\{Z, \rho\}$ снова положительна, а значит, покрывается другим листом. Нетрудно убедиться, что прямые, проходящие в точку $Z=0, P=0$ (т. е. в точку $\gamma=0$ ), отражаются на этом втором листе обратно по той же прямой. Это значит, что геометрически удобно устроить отражение векторов на втором листе с помощью матрицы $-I$, где $I$ - двумерная единичная матрица, т. е. зеркально отразить лист $\gamma<0$ в отрицательный квадрант.

Отметим, что эта процедура соответствует концепции дырок Дирака, только наоборот - дыркам мы ставим в соответствие отрицательное давление, т. е. отрицательную энергию. Теперь прямые будут продолжаться через точку начала координат в отрицательный квадрант, хотя давление реально не меняет знак. Это лишь удобная геометрическая "униформизация".

Отметим также, что ввиду отсутствия притяжения идеальная жидкость полностью пластична: она не стремится возвратиться в исходное (до растяжения) состояние. В этом смысле бозе-конденсат при $\gamma<0$, приводящий к этому “виду" жидкости, также более наглядно может быть воспринят как стекло или аморфное тело. Это состояние жидкости при $\gamma<0$ более наглядно.

ЗАмЕчАниЕ 3. Автор пришел к пересмотру термодинамики при изучении процессов в экономике, в которой согласно принципу соответствия, выведенному Ирвингом Фишером, деньги - это частицы. Фишер сам не назвал свое наблюдение принципом соответствия. Но поскольку он был учеником Гиббса, то соотношение основного закона экономики

$$
P Q=M v
$$

где $Q$ - объем товаров, $M$ - число денег, $v$ - скорость оборота, а $P$ - цена товаров, очевидным образом связано с соответствием экономических и термодинамических величин: объем товаров $Q$ - объем $V$, число денег $M$ - число частиц $N$, скорость 
оборота $v$ - температура $T$. Цена товаров $P$ не в такой степени связана с давлением, зато обозначается той же буквой.

В этом принципе соответствия естественно сопоставить дырки долгам, погашение долгов - аннигиляции.

Как было сказано выше, геометрическое место точек, в котором химический потенциал равен нулю, - это точки максимальной энтропии. Мы их назвали “новой спинодалью". В экономике эта новая спинодаль означает некий предел долгов.

Итак, согласно полученным соотношениям мы получаем двулистное покрытие плоскости $\{Z, P\}$ при $\gamma \geqslant 0$ и $-1 \leqslant \gamma<0$. Смысл второго листа заключается в том, что при $-1 \leqslant \gamma<0$ хаотическое состояние жидкости, как явления, связанного с бозе-конденсатом, увеличивается, когда число дырок типа экситонов Френкеля увеличивается, располагаясь в жидкости, как флуктуационно сосредоточенной на сравнительно малоподвижной, с точки зрения вышеуказанного прибора ${ }^{2)}$, области возникает хаотические нанодырки - структура жидкости хаотически растягивается.

Дырки-экситоны при этом не могут быть пронумерованы нашим прибором, как и частицы, и мы можем говорить лишь о плотности дырок. Этот второй лист для удобства лучше расположить в квадранте $[-Z,-P]$, продолжая прямые (16) за особую точку $Z=0, P=0$ в отрицательный квадрант. Иначе говоря, совершить отражение с помощью матрицы $-I$, где $I$ - единичная матрица.

Тем самым оказывается удобно говорить об "отрицательном давлении”, хотя притяжением частиц мы пренебрегаем и, значит, никакого отрицательного давления быть не может. Давление, как и температура, обычно считается положительным. Мы растягиваем жидкость, а она пластически застывает в этом растянутом состоянии и не стремится сжаться обратно.

Таким образом, более наглядно и геометрически удобно говорить об “отрицательном давлении", тогда как на самом деле это давление связано с наличием дырок в бозе-конденсате. Новая спинодаль как совокупность точек, в которых энтропия жидкости при данной температуре максимальна, экспериментально достигается равновесным растяжением жидкости. Растяжение пластической структуры обычно не называют отрицательным давлением, но в нашем случае, как уже говорилось, подобно теории дырок Дирака, удобно рассматривать вместо дырок отрицательную энергию (т. е. давление), хотя, в отличие от уравнения Дирака, для любого вещества, как мы увидим, “отрицательная энергия" не простирается до минус бесконечности, а ограничена по модулю. В этом смысле можно было бы сдвинуть начало отсчета для энергии, как это обычно делается в теории полуопределенных операторов. Однако если потенциал на бесконечности равен константе, то эту константу принято считать за начало отсчета энергии (например, для потенциала Леннарда-Джонса). В теории Ван-дер-Ваальса отрицательное давление простирается до бесконечности, но это физически недопустимо.

Пренебрежение наличием притяжения здесь столь же "законно", как в теории равновесия пар-жидкость, где условие равенства давлений возможно лишь при пренебрежении поверхностным натяжением.

${ }^{2)}$ В реальности дырки могут быстро и незаметно для глаз и прибора меняться между собой и окружающим газом. 
Этим объясняется и гладкий переход (без фазового скачка первого рода) этой структуры в лед - застывшее стекло кристаллизуется.

Перейдем к вычислению максимальной энтропии на изотермах жидкой фазы, определенной с учетом Zeno line.

\section{5. УЧЕТ ПРИТЯЖЕНИЯ И ZENO LINE}

Притяжение между частицами возникает в квантово-механическом рассмотрении диполь-дипольного взаимодействия. В стандартном квазиклассическом пределе, если расстояние между нейтральными молекулами фиксировано (не зависит от параметра $\hbar$, т. е. от безразмерного параметра, пропорционального $\hbar)$, при $\hbar \rightarrow 0$ притяжение исчезает. В этом смысле использование притягивающего потенциала в молекулярной динамике, опирающейся на классические уравнения Ньютона для многих частиц, необоснованно.

Однако если в задаче кроме $\hbar$ участвуют другие малые и большие параметры, то при некоторых соотношениях между ними при $\hbar \rightarrow 0$ притягивающий потенциал может сохраняться. Например, это возможно в коротковолновом приближении при условии Зоммерфельда на бесконечности, приводящем к несамосопряженной задаче и учитывающем, по существу, исчезающе малую вязкость.

В квантовой задаче рассеяния при учете диссипации и задаче, строго изученной после работы Куроды [17], квазиклассический предел сохраняет потенциал притяжения, как говорят физики, “для ближайшей соседки". Не останавливаясь на этих достаточно сложных оценках, рассмотрим классическую задачу рассеяния, содержащую притягивающий потенциал, в частности потенциал Леннарда-Джонса.

Как известно, в радиально-симметричном случае

$$
\frac{m v^{2}}{2}+\frac{M^{2}}{2 m r^{2}}+\Phi(r)=E .
$$

Для исходных рассеивающихся частиц задаются энергия $E$ и прицельный параметр $B$. Момент $M$, как и энергия $E$, сохраняется. Известно также, что

$$
M^{2}=B^{2} E \text {. }
$$

Выразив энергию $E$, получим для притяжения

$$
E=\frac{\left(m v^{2}\right) / 2+\Phi(r)}{1-B^{2} / r^{2}}
$$

в области, где $r \leqslant B$.

На рис. 6 мы приводим диаграмму, отвечающую современному состоянию термодинамики, полученную как с помощью методов молекулярной динамики (компьютерных экспериментов), так и с помощью физических экспериментов.

Современная термодинамика опирается не на известное эмпирическое соотношение Ван-дер-Ваальса, а в основном на результаты компьютерного эксперимента в области новой науки, которая носит название “молекулярная динамика". Результаты этого эксперимента приведены на рис. 6. Сплошная прямая, исходящая из точки Бойля, называется Zeno line, а штриховая прямая называется “бинодаль" (т. е. кривая, разделяющая две фазы) [18]. При заданной температуре, меньшей $T_{\mathrm{c}}$, происходит скачок плотности $\rho_{\mathrm{r}}$, отложенной по оси абсцисс. 


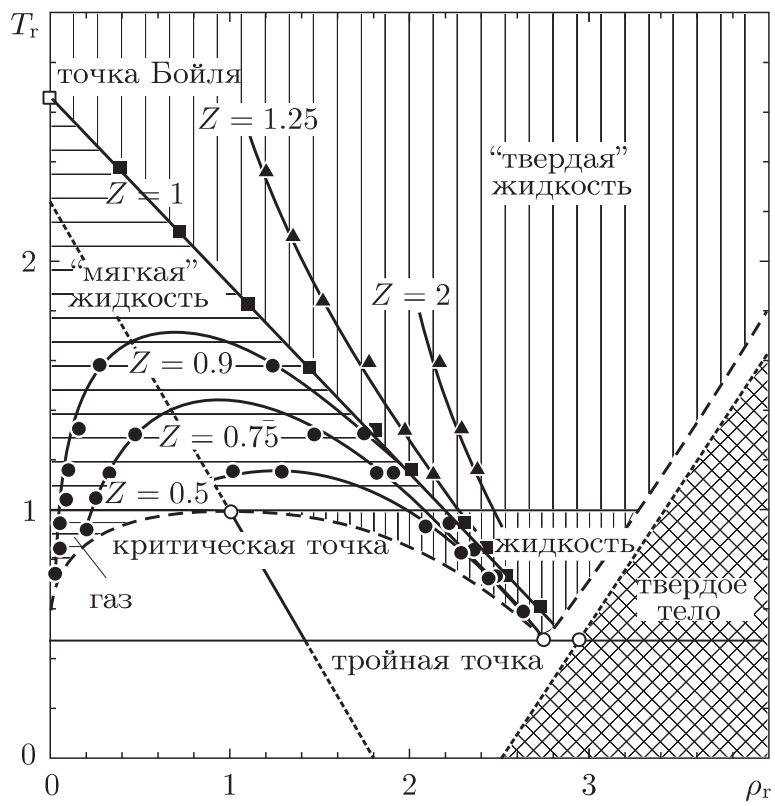

Рис. 6. Диаграмма $\left(T_{\mathrm{r}}, \rho_{\mathrm{r}}\right)$ для газов, соответствующих простым жидкостям, $T_{\mathrm{r}}=T / T_{\mathrm{c}}, \rho_{\mathrm{r}}=\rho / \rho_{\mathrm{c}}$. Zeno line (прямая $\left.Z=P_{\mathrm{r}} V / k N T_{\mathrm{r}}=1.0\right)$ на фазовой диаграмме. Для состояний $Z>1.0$ доминируют силы отталкивания, для состояний $Z<1.0$ - силы притяжения.

В задаче рассеяния в качестве потенциала взаимодействия рассматривают потенциал Леннарда-Джонса

$$
\Phi\left(r^{\prime}, r^{\prime \prime}\right)=4 \varepsilon\left(\frac{a^{12}}{\left\|r^{\prime}-r^{\prime \prime}\right\|^{12}}-\frac{a^{6}}{\left\|r^{\prime}-r^{\prime \prime}\right\|^{6}}\right), \quad r=r^{\prime}-r^{\prime \prime},
$$

где $\varepsilon$ - энергия глубины ямы, $a$ - эффективный радиус, $\left\|r^{\prime}-r^{\prime \prime}\right\|$ - расстояние между двумя частицами с радиус-векторами $r^{\prime}, r^{\prime \prime}$. В двухчастичной задаче при отсутствии внешнего потенциала задача приводится к одномерной радиально-симметричной.

В задаче (29) при различных значениях $B$ возникают другие барьеры и ямки (рис. 7,8$)$. В точках покоя $E_{\min }$ и $E_{\max }$ скорость равна нулю, поэтому они могут быть рассчитаны только по потенциальному члену.

Речь теперь идет не об одной частице, а о паре частиц с центром масс, который попадает в ловушку. Поэтому разность $E_{\max }-E_{\min }-$ это та энергия, с которой можно выбить эту пару (димер) из ловушки. В эксперименте можно определить процент димеров в газе. Видно, как димеры рождаются и разбиваются мономерами. Затем подсчитывается их среднее число. Чем выше температура, тем больше средняя энергия мономеров и тем меньше число димеров.

Главное, что при таком подходе у нас остается в скелете задачи рассеяния (ср. скелет амебы в тропической математике [19]) только две величины $E_{\max }$ и $E_{\min }$. Когда $E_{\max }=E_{\min }$, ямка исчезает. Для притягивающей части потенциала Леннарда-Джонса эта энергия равна $0.8 \varepsilon$. С учетом вышеприведенной изотропии получаем, 


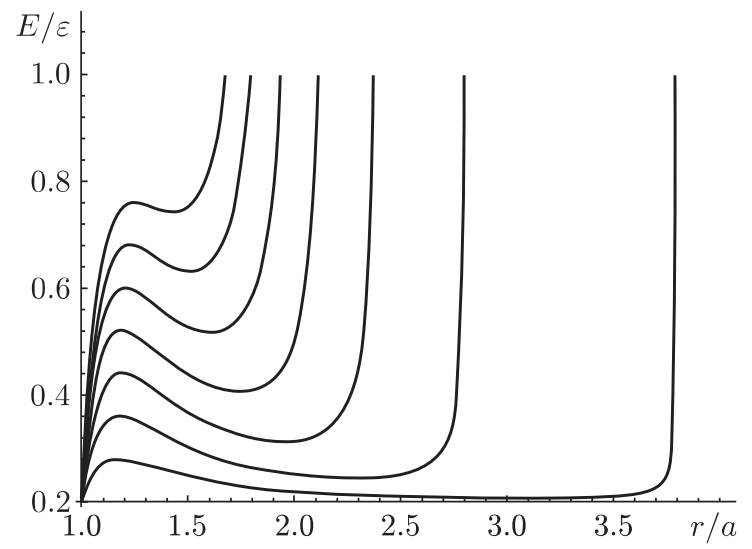

Рис. 7. Ямки и барьеры в задаче рассеяния двух частиц с потенциалом взаимодействия Леннарда-Джонса при различных прицельных параметрах $B$.

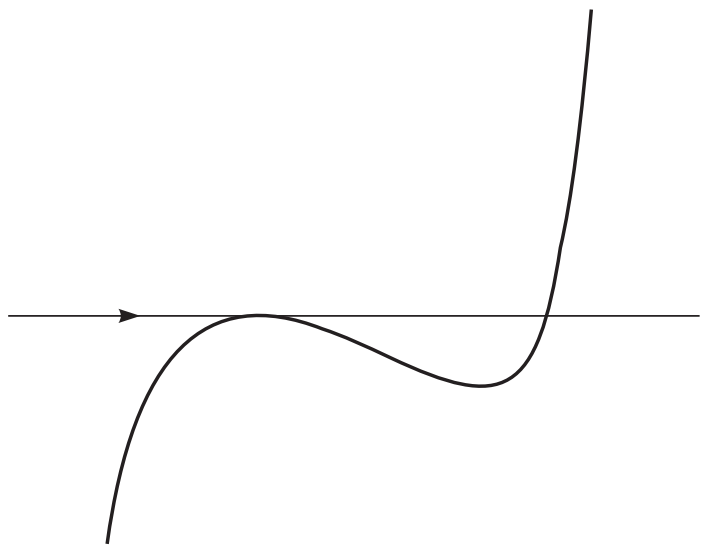

Рис. 8. Ловушка для фиктивной частицы (димера) в системе центра масс; $r$ - радиус-вектор димера расположен по оси абсцисс. Частица падает слева из точки $r=B$.

что средняя энергия частиц равна $16 \varepsilon / 5$. Средняя энергия соответствует температуре $T=16 \varepsilon / 5 k$. Выше этой температуры ямка отсутствует. В термодинамике это так называемая температура Бойля $T_{\mathrm{B}}$. Согласно данным о величине $\varepsilon$ по этой формуле температура Бойля для аргона $(\mathrm{Ar}) T_{\mathrm{B}}=382 \mathrm{~K}$, для крептона $(\mathrm{Kr}) T_{\mathrm{B}}=547 \mathrm{~K}$, а по экспериментальным таблицам работы [20] для аргона $T_{\mathrm{B}}=392 \mathrm{~K}$, для крептона $T_{\mathrm{B}}=538 \mathrm{~K}$. Расхождение теоретических значений с экспериментальными составляет $2-3 \%$.

Критическая температура $E_{\max }$ должна отвечать самой глубокой ямке - максимальному значению разности $E_{\max }-E_{\min }$ для всех прицельных параметров $B$. Эта разность определяет, насколько упала энергия димера после его захвата "ловушкой" и, тем самым, какую энергию нужно иметь мономеру, чтобы димер выскочил из ямы (т. е. распался).

5 Теоретическая и математическая физика, т. 175, № 1, 2013 г. 
Высота барьера "защищает" возникшую пару, приведенная масса которой попала в ловушку “димера" и кластеров, от “ударов" мономеров. При уменьшении тем-

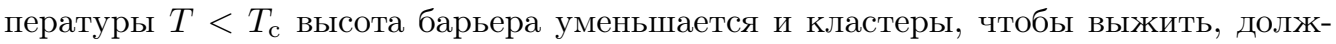
ны создать собственный барьер в виде микроскопического аналога поверхностной пленки. Значит, должен появиться “домен” - трехмерный кластер (так называемый элементарный кластер), у которого существует хотя бы одна частица, защищенная другими частицами.

Вычисления дают $E_{\max }=0.286 \varepsilon / k$ в точке $\max _{B}\left(E_{\max }-E_{\min }\right)$. Прицельный параметр в этой точке равен $B=2.436$.

ЗАмЕчАниЕ 4. Определение критической температуры и температуры Бойля через глубину ямы потенциала взаимодействия $\varepsilon$ носит весьма неточный характер, и в разных справочниках приводятся разные данные. Поэтому мы в дальнейшем будем рассматривать безразмерную величину $T_{\mathrm{B}} / T_{\mathrm{c}}=2.79$, что лучше согласуется с экспериментальными данными (для аргона это значение равно 2.73).

Единственная безразмерная величина в нашей "усушенной” до скелета задаче является $E_{\min } / E_{\max }$, т. е. в задаче, в которой мы оставили только ее скелет, имеются две точки покоя.

Поскольку $E_{\min } / E_{\max }$ - безразмерная величина, а $E_{\max }$ как среднюю энергию мы связываем с температурой (умноженной на плотность $\rho$ ), то безразмерной величиной в термодинамике является так называемый фактор сжимаемости, обозначаемый буквой $Z: Z=P V / R T$.

Теперь получим аналитические формулы для Zeno line в зависимости от потенциала.

Исходное допущение заключается в том, что термодинамическое описание не зависит от формы сосуда. Поскольку для термодинамических величин важен только объем, а не его форма, то мы можем по значению объема найти характерную длину. Для сосуда в виде шара такой величиной является радиус шара, а для узкой длинной пробирки, использованной, например, Торричелли, - радиус пробирки. Поэтому в общем случае, согласно теории размерностей у нас имеется объемная мера, а мера длины есть эффективный радиус $a$.

Используем соображения теории размерностей для задачи рассеяния и определения притягивающего одночастичного (так называемого термического) потенциала. Задача рассеяния рассматривается для потенциала Леннарда-Джонса, и, следовательно, в задаче имеется дополнительный параметр длины $a$ - эффективный радиус. Притягивающий потенциал возникает в квантовой теории диполь-дипольного взаимодействия. Если фиксировать расстояние между частицами и квазиклассический параметр $\hbar$ устремить к нулю, то притягивающий потенциал обратится в нуль. Это значит, что при $\hbar \rightarrow 0$ расстояние между частицами уменьшается. Отсюда следует, что:

1) использовать притягивающий потенциал в задаче молекулярной динамики для классической системы уравнений Ньютона по меньшей мере некорректно;

2) притягивающий потенциал действует лишь между ближайшими соседями.

Таким образом, разложение притягивающего одночастичного потенциала по степеням радиуса $r$ естественно брать с точностью до $O\left(r^{3} / V\right)$. 
"Термический" потенциал $\Psi(r)$ является притягивающим. Кроме того, поскольку объем $V$ является большим параметром, то разложение потенциала $\Psi(r)=\Psi\left(a r^{2} / V\right)$ по $1 / V$ дает

$$
\Psi\left(\frac{a r^{2}}{V}\right)=C_{1}+\frac{C_{2} a r^{2}}{V}+O\left(\frac{1}{V^{2}}\right)
$$

Разлагая

$$
r^{2}=r_{1}^{2}+r_{2}^{2}=\frac{\left(r_{1}-r_{2}\right)^{2}}{2}+\frac{\left(r_{1}+r_{2}\right)^{2}}{2}
$$

мы можем, подобно тому, как это было сделано в [21], произвести разделение переменных в двухчастичной задаче и перейти к задаче рассеяния для пары частиц и к задаче их совместного (для $r_{1}+r_{2}$ ) движения. Тогда в задаче рассеяния к потенциалу взаимодействия Леннарда-Джонса добавится притягивающий квадратичный потенциал (перевернутая парабола): $-C_{2} \rho r^{2}, \rho=a / V$.

Наличие термического потенциала не следует из всей предыдущей и последующей конструкции. Он может быть получен из идей самосогласованного поля Власова-Боголюбова или конечности корреляционной сферы для $N$-частичного распределения Гиббса. Как мы увидим далее, зависимость от плотности дает существенное дополнение к распределению теории чисел (см., например, [21], раздел 3).

В полученной таким образом задаче рассеяния, как правило, имеются две точки покоя - устойчивая $E_{\min }$ и неустойчивая $E_{\max }$. Отношение этих величин является безразмерной величиной. В термодинамике безразмерной величиной является $Z=$ $P V / N T$, и в силу того, что устойчивая точка покоя по смыслу отвечает температуре, то соотношение

$$
Z=\frac{P V}{N T}=\frac{E_{\min }}{E_{\max }}
$$

позволяет построить на графике $(T, \rho=N / V)$ кривые $Z=$ const.

Кривая при $Z=1$ называется Zeno line (или парабола Бачинского), а геометрическое место точек начала кривых при $Z=$ const (при $C_{2} \neq 0, B \rightarrow \infty$ ) - бинодалью.

Минимальное значение фактора сжимаемости $Z$ для данного $\rho$ при $C_{2} \neq 0$ равно $Z_{\min }=E_{\min } /\left.E_{\max }\right|_{B \rightarrow \infty}$. При $Z_{\min }=0.8 \varepsilon$ и $\rho=0$ оно соответствует температуре Бойля. Как известно, точка на бинодали, соответствующая $Z_{\min }$ для значения $\rho=0$, отвечает абсолютному нулю.

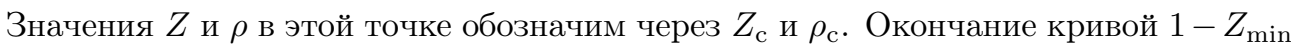
на оси $\rho$ обозначим $\rho_{\mathrm{B}}$. В работе [22] эту точку назвали гипотетической точкой Бойля.

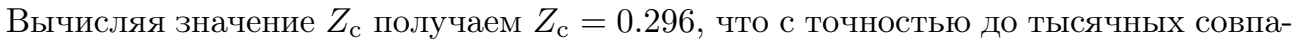

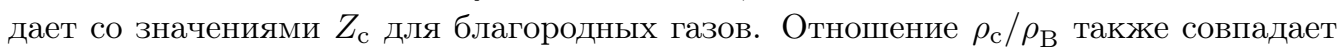
со значениями этой величины для благородных газов.

В табл. 1 приведены данные, отвечающие полученной диаграмме (для $B=100$ в "молекулярных" значениях). Отметим расхождение основных безразмерных соотношений, полученных по данными молекулярной динамики, с теоретическими 
соотношениями, полученными физиками из цепочки ББГКИ и $n$-частичного распределения Гиббса.

ТАБЛИцА 1

\begin{tabular}{|c|c|c|}
\hline$Z_{\mathrm{c}}$ & $\rho_{\mathrm{c}} / \rho_{\mathrm{B}}$ & $T_{\mathrm{c}} / T_{\mathrm{B}}$ \\
\hline 0.29 & 0.273 & 0.36 \\
\hline 0.308 & 0.285 & 0.38 \\
\hline 0.375 & 0.333 & 0.296 \\
\hline
\end{tabular}

В первой строке таблицы указаны теоретические значения для $Z_{\mathrm{c}}, \rho_{\mathrm{c}} / \rho_{\mathrm{B}}$ и $T_{\mathrm{c}} / T_{\mathrm{B}}$, полученные с помощью соображений теории размерностей, приведенных выше, во второй строке - значения этих же величин по последним данным молекулярной динамики и физиков-теоретиков для потенциала Леннарда-Джонса, в третьей строке значения, полученные из уравнения Ван-дер-Ваальса, которое является эмпирическим.

Значение $Z_{\text {с }}$ очень точно считается на эксперименте, оно равно 0.29 для благородных газов, азота, кислорода, пропана. Значение $\rho_{\mathrm{c}} / \rho_{\mathrm{B}}$ (т. е. отношение $\rho_{\mathrm{c}}$ ко всей длине отрезка по $\rho$, где Zeno line "отрезает" ось абсцисс) совпадает с соответствующими значениями для воды, аргона, ксенона, криптона, этилена и ряда других газов.

Теперь построим диаграмму $(Z, \rho)$. Мы строим на этой диаграмме кривую $Z=$ $1-\left.\left(E_{\min } / E_{\max }\right)\right|_{B=\max }$ и прямую, соединяющую ее конечные точки $(Z=1$ и $Z=0)$. Если термический потенциал присутствует, то $B \rightarrow \infty$ и кривая имеет вид, изображенный на рис. 9.

Критическую температуру мы определили для "чистого" потенциала Леннарда-

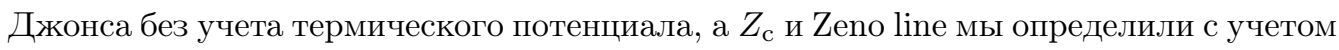
термического потенциала. Для определения критической температуры мы рассмотрели максимальную глубину ямки $E_{\max }-E_{\min }$ для “чистого" потенциала ЛеннардаДжонса.

Приведем подробные вычисления для определения Zeno line. Рассмотрим потенциал

$$
E(r)=\frac{-\alpha r^{4}+r^{2} U(r)}{B^{2}-r^{2}} .
$$

Его первая и вторая производные равны

$$
\begin{aligned}
E^{\prime}(r)= & \frac{r\left(2 B^{2} U(r)+r\left(2 \alpha r\left(-2 B^{2}+r^{2}\right)+\left(B^{2}-r^{2}\right) U^{\prime}(r)\right)\right)}{\left(B^{2}-r^{2}\right)^{2}}, \\
E^{\prime \prime}(R)= & \frac{1}{\left(B^{2}-r^{2}\right)^{3}}\left(2\left(B^{4}+3 B^{2} r^{2}\right) U(r)+r\left(-2 \alpha r\left(6 B^{4}-3 B^{2} r^{2}+r^{4}\right)+\right.\right. \\
& \left.\left.+4\left(B^{4}-B^{2} r^{2}\right) U^{\prime}(r)+r^{2}\left(B^{2}-r^{2}\right)^{2} U^{\prime \prime}(r)\right)\right) .
\end{aligned}
$$

Получаем решение уравнения в виде

$$
B=\sqrt{-\frac{-r^{3} U^{\prime}(r)+r^{4} U^{\prime \prime}(r)}{-8 U(r)+2 r U^{\prime}(r)+2 r^{2} U^{\prime \prime}(r)}} .
$$




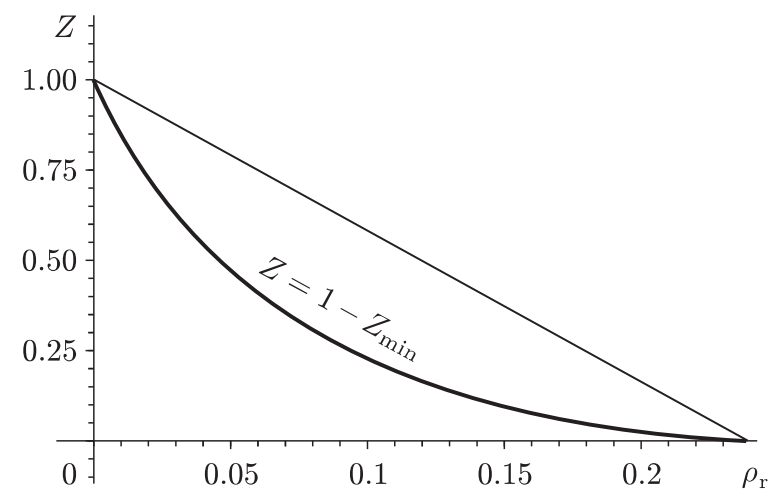

Рис. 9. Кривая $1-Z_{\min }$.

Подставляя значение $B(r)$ в $(34)$, находим $E(\alpha)-$ Zeno line. Затем, полагая $B=100$, находим значения первой производной. Приравниваем ее нулю и находим отношение $\left(E_{\max }-E_{\min }\right) / E_{\max }$.

\section{6. ОПРЕДЕЛЕНИЕ НОВОЙ КРИТИЧЕСКОЙ ТОЧКИ}

Имеем

$$
Z=\frac{P_{\mathrm{r}}}{\rho T_{\mathrm{r}}}
$$

где

$$
\rho=\rho_{\mathrm{B}}\left(1-\frac{T_{\mathrm{r}}}{T_{\mathrm{B}}}\right)
$$

- изотерма-изохора. Поэтому

$$
Z=\frac{P_{\mathrm{r}}}{\rho_{\mathrm{B}} T_{\mathrm{r}}\left(1-T_{\mathrm{r}} / T_{\mathrm{B}}\right)} .
$$

Выразим $Z$ через $\gamma$ при $Z<0, \gamma<0$ и при $\mu \rightarrow 0$ :

$$
N=A(\gamma) T_{\mathrm{r}}, \quad P=\frac{T_{\mathrm{r}}^{2+\gamma} \zeta(2+\gamma)}{\zeta\left(2+\gamma_{\mathrm{c}}\right)} .
$$

(Здесь и ниже значение $\gamma_{\mathrm{c}} \sim 0.1$ отвечает нижнему значению $T_{\mathrm{r}}=1$ на прямой $P_{\mathrm{r}}=1$ согласно рис. $10 ;$ см. раздел 9.)

Значение $\rho_{\mathrm{B}}$ определяется в этой нормировке. Таким образом, получим

$$
Z=\frac{T_{\mathrm{r}}^{2+\gamma} \zeta(2+\gamma)}{\zeta\left(2+\gamma_{\mathrm{c}}\right) A(\gamma) T^{2}}=\frac{T_{\mathrm{r}}^{\gamma} \zeta(2+\gamma)}{\zeta\left(2+\gamma_{\mathrm{c}}\right) A(\gamma)}
$$

С другой стороны,

$$
N=\rho=\rho_{\mathrm{B}}\left(1-\frac{T_{\mathrm{r}}}{T_{\mathrm{B}}}\right)=A(\gamma) T_{\mathrm{r}}
$$




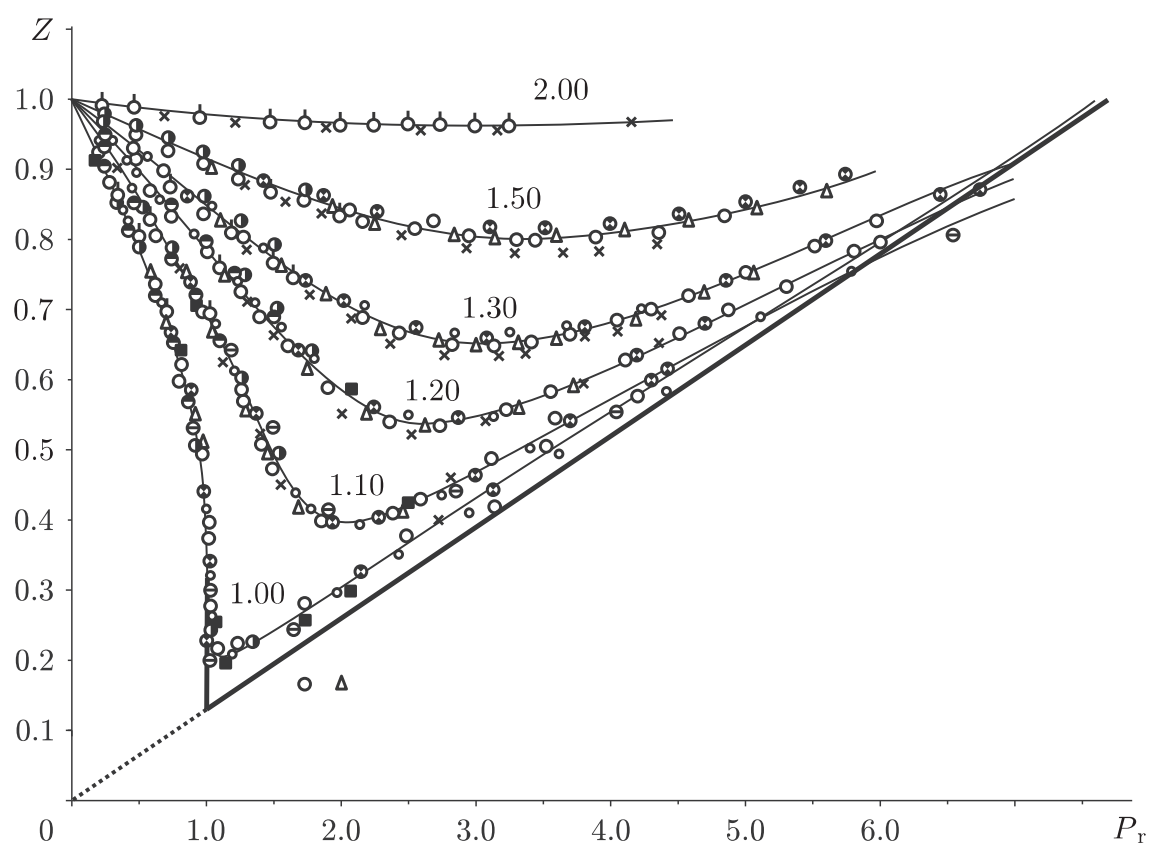

Рис. 10. Картина закона соответственных состояний для молекул различных газов. Тонкие линии изображают изотермы метана. Разные символы на изотермах соответствуют аргону, углекислому газу, воде и др. Близость изотерм различных газов иллюстрирует эмпирический закон соответственных

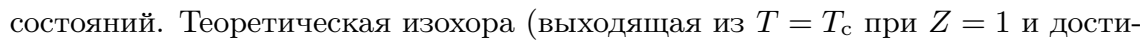
гающая начало координат линия) не полностью совпадает с экспериментальной. Это эффект того же типа, что и скачок критических показателей. Вязкость (винеровское квантование) сглаживает острый угол предельной (при $\varepsilon \rightarrow 0)$ изотермы. Значение $\gamma_{c}=0.1$ отвечает угловой точке.

Следовательно,

$$
\begin{gathered}
\frac{\rho_{\mathrm{B}}}{T_{\mathrm{r}}}-\frac{\rho_{\mathrm{B}}}{T_{\mathrm{B}}}=A(\gamma), \\
\left(\frac{1}{T_{\mathrm{r}}}\right)^{|\gamma|}=\left(\frac{1}{\rho_{\mathrm{B}}} A(\gamma)+\frac{1}{T_{\mathrm{B}}}\right)^{|\gamma|} .
\end{gathered}
$$

Подставляя последнее выражение в (39), получаем

$$
Z=\frac{\zeta(\gamma+2)}{\zeta\left(\gamma_{\mathrm{c}}+2\right)} \frac{\left(A(\gamma) / \rho_{\mathrm{B}}+1 / T_{\mathrm{B}}\right)^{|\gamma|}}{A(\gamma)}
$$

при $\gamma \rightarrow-1, A(\gamma) \rightarrow \infty$.

При $\gamma \rightarrow-1$ числом $1 / T_{\mathrm{B}}$ можно пренебречь. Следовательно,

$$
\left.Z\right|_{\gamma \rightarrow-1} \simeq \frac{\zeta(\gamma+2)}{\rho_{\mathrm{B}}^{|\gamma|} \zeta\left(\gamma_{\mathrm{c}}+2\right) A(\gamma)^{1-|\gamma|}} .
$$


Поскольку

$$
A(\gamma, \lambda)=\left(\lambda^{\gamma-\gamma_{c}} c(\gamma)\right)^{1 /(1+\gamma)}
$$

где $\gamma<0$, то

$$
A(\gamma, \lambda)^{1+\gamma}=\lambda^{\gamma-\gamma_{c}} c(\gamma)
$$

где

$$
c(\gamma)=\left[\int_{0}^{\infty} t^{\gamma} d t\left(\frac{1}{t}-\frac{1}{e^{t}-1}\right)\right] .
$$

Следовательно,

$$
\left.Z\right|_{\gamma \rightarrow-1}=\frac{\zeta(2+\gamma) \lambda^{\gamma_{\mathrm{c}}+1}}{\rho_{\mathrm{B}}^{|\gamma|} \zeta\left(2+\gamma_{\mathrm{c}}\right) c(\gamma)} .
$$

Выражение $c(\gamma)$ при $\gamma \rightarrow-1$ стремится к $(1 / 2) \ln \varepsilon$, где $\varepsilon-$ нижний предел интегрального выражения для $A(\gamma)^{1-|\gamma|}$ при $\gamma=-1$. Аналогично выражение $\zeta(\gamma+2)$ при $\gamma=-1$ равно $\ln \varepsilon$. Отсюда получаем

$$
\left.Z\right|_{\gamma \rightarrow-1}=\frac{2 \lambda^{\gamma_{c}+1}}{\rho_{\mathrm{B}} \zeta\left(\gamma_{\mathrm{c}}+2\right)}
$$

при $\gamma \rightarrow 0, Z \rightarrow 0$. Поэтому $Z<Z_{\max }<$ const при всех значениях $\gamma$.

Отсюда в силу (38) имеем

$$
P_{\mathrm{r}}=Z \rho_{\mathrm{B}} T_{\mathrm{r}}\left(1-\frac{T_{\mathrm{r}}}{T_{\mathrm{B}}}\right) \leqslant Z_{\max } \frac{T_{\mathrm{B}}}{2} .
$$

Кроме того, очевидно, что $P_{\mathrm{r}} \rightarrow 0$ при $T_{\mathrm{r}} \rightarrow 0$ и при $\gamma \rightarrow 0$ (см. рис. 10).

Величина $\lambda$ определяет минимум $P_{\mathrm{r}}$. С другой стороны, значение $\lambda$ мы вычисляем, если известна величина тройной точки на бинодали. Таким образом, величина $\min P_{\mathrm{r}}$, которая соответствует новой критической точке (см. рис. 11) в термодинамике перехода жидкостей в дисперсную фазу, может быть определена однозначно через значение критической точки $Z_{\mathrm{c}}$, Zeno line и тройную точку.

Для определения перехода газ-жидкость, как и в разделе 3 , с учетом поправки на Zeno line мы выводим уравнения типа уравнений (24), (25), производя для критической изотермы нормировку активности.

\section{7. ФАЗОВЫЙ ПЕРЕХОД В ИДЕАЛЬНОМ ГАЗЕ БОЛЬЦМАНА-МАКСВЕЛЛА}

Чем меньше температура, тем больше газа (насыщенного пара) перейдет в жидкость. Следовательно, газ будет становиться более и более разреженным при уменьшении температуры. Для разреженного газа в атмосфере известен так называемый критерий Кнудсена. Он возникает, когда длина свободного пробега становится соизмеримой с характерной длиной объекта (спутника, самолета), который находится в атмосфере.

В нашем случае газ находится внутри "объекта" - граница объема $V$ является ловушкой, которая не выпускает газ наружу. При этом геометрией границы объема $V$ мы принципиально пренебрегаем. Имеется достаточно большой объем $V$, и задача должна быть инварианта относительно изменения его формы. Внешнего 


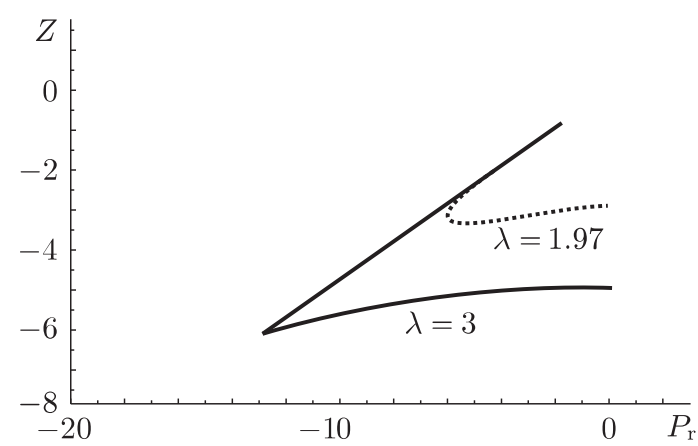

Рис. 11. Кривые спинодали в области $P_{\mathrm{r}}<0$. Наклонная прямая - продолжение теоретической критической изотермы с рис. 10 в область отрицательных $P_{\text {r }}$ и $Z$. Начальная точка кривой $\lambda=3$ отстоит от точки $P_{\mathrm{r}}=0$, a начальная точка $\lambda=1.97$ кривой совпадает с точкой пересечения пунктирной кривой и сплошной линии.

поля в нашей задаче нет, поэтому одночастичный потенциал в распределении Гиббса (в гиббсовском ансамбле, см. [23]) должен быть представлен при бесконечно малом радиусе молекул в виде $\varphi\left(r^{3} / V\right), \varphi \in C^{\infty}$. А так как объем велик, то главный член имеет вид $-\alpha r^{3} / V$, где $\alpha$ - параметр, который выражается, как мы увидим ниже, через параметр $a$. Эта зависимость возникает потому, что ввиду инвариантности относительно формы объема $V$ линейного параметра по координатам в макроразмерности у нас нет.

Поскольку дополнительные параметры не должны зависеть от формы сосуда, то для их определения достаточно взять объем в виде шара радиуса $R$.

Мы заменим параметр целого числа степеней свободы некоторым непрерывным параметром, который характеризует данную молекулу. В принципе этот параметр $\gamma$ имеет то же физическое происхождение, что и число степеней свободы. Но поскольку он непрерывный, то более тонко учитывает спектр молекулы.

Безразмерный параметр $\lambda$ обратно пропорционален массе. Несущественно, с помощью каких еще величин мы "обезразмериваем" массу.

Энергия имеет вид

$$
\mathcal{E}_{\mathrm{r}}=\frac{p^{2}}{2 m}+C \frac{r^{3}}{R^{3}} .
$$

Согласно интегралу от распределения Гиббса получаем

$$
\int_{R_{1}}^{R} \int_{|p| \geqslant 0} \exp \left\{\frac{-p^{2} / 2 m-C r^{3} / R^{3}+\mu}{T_{\mathrm{r}}}\right\} d r^{3} d|p|^{3},
$$

где $R_{1}=R\left(1-\varepsilon\left(1-T_{\mathrm{r}}^{1 / 2}\right)\right)$, параметр $\varepsilon$ - аналог числа Кнудсена, что приводит к закону Клапейрона-Клаузиуса при $T \rightarrow 0$. Зависимость от температуры области влияния границы выбирается так, чтобы на диаграмме $\left(Z, P_{\mathrm{r}}\right)$ на газовой изотерме вблизи спинодали сохранялось не только условие $d P_{\mathrm{r}} / d Z=\infty$, но и порядок обращения в бесконечность производной. 
Если пренебречь влиянием стенок сосуда, то изотерма идеального газа в плоскости $\left\{P_{\mathrm{r}}, Z_{\mathrm{r}}\right\}$ имеет вид

$$
P_{\mathrm{r}}=T_{\mathrm{r}}^{2+\gamma_{\mathrm{c}}} \frac{\operatorname{Li}_{2+\gamma_{\mathrm{c}}}(a)}{\zeta\left(2+\gamma_{\mathrm{c}}\right)}, \quad Z=\frac{\operatorname{Li}_{2+\gamma_{\mathrm{c}}}(a)}{\operatorname{Li}_{1+\gamma_{\mathrm{c}}}(a)}, \quad a=e^{-\mu / T}, \quad \mu \leqslant 0
$$

(см. графики в работе [24]).

Учет потенциала $r^{3} / V$ изменяет давление и фактор сжимаемости $Z$. Это позволяет учесть в общем виде отражение от стенок сосуда для нового идеального газа. Имеем

$$
\int_{R_{1}}^{R} e^{-r^{3} C k / R^{3}} d r^{3}=\frac{R^{3}}{C k} \int_{k C R_{1} / R}^{k C} e^{-x} d x
$$

Отсюда $k$-й член в определении полилогарифма для бозе-распределения с дробным числом степеней свободы

$$
\operatorname{Li}_{s}(a)=\sum_{k=1}^{\infty} \frac{a^{k}}{k^{s}}
$$

принимает вид

$$
\frac{e^{C k / T_{\mathrm{r}}}-e^{\left(C k / T_{\mathrm{r}}\right)\left(1-\varepsilon\left(1-T_{\mathrm{r}}^{1 / 2}\right)\right)}}{C k^{3+\gamma_{\mathrm{c}}}} .
$$

Отсюда получаем

$$
\begin{gathered}
P=-\frac{T_{\mathrm{r}}^{3+\gamma_{\mathrm{c}}}}{C}\left\{\operatorname{Li}_{3+\gamma_{\mathrm{c}}}\left(a e^{-C / T_{\mathrm{r}}}\right)-\operatorname{Li}_{3+\gamma_{\mathrm{c}}}\left(a e^{-C / T_{\mathrm{r}}\left\{1-\varepsilon\left(1-T_{\mathrm{r}}^{1 / 2}\right)\right\}}\right)\right\} \cdot \text { const, } \\
Z\left(a, T_{\mathrm{r}}\right)=\frac{\operatorname{Li}_{3+\gamma_{\mathrm{c}}}\left(a e^{-C / T_{\mathrm{r}}}\right)-\mathrm{Li}_{3+\gamma_{\mathrm{c}}}\left(a e^{-C / T_{\mathrm{r}}\left\{1-\varepsilon\left(1-T_{\mathrm{r}}^{1 / 2}\right)\right\}}\right)}{\operatorname{Li}_{2+\gamma_{\mathrm{c}}}\left(a e^{-C / T_{\mathrm{r}}}\right)-\mathrm{Li}_{2+\gamma_{\mathrm{c}}}\left(a e^{-C / T_{\mathrm{r}}\left\{1-\varepsilon\left(1-T_{\mathrm{r}}^{1 / 2}\right)\right\}}\right)} .
\end{gathered}
$$

Заметим, что $Z\left(1, T_{\mathrm{c}}\right)=Z_{\mathrm{c}}$. Константа в (45) выбирается из условия нормировки Ван-дер-Ваальса: $T_{\mathrm{r}}=T_{\mathrm{r}} / T_{\mathrm{c}}, P_{\mathrm{r}}=P / P_{\mathrm{c}}$. При этом в определении давления отброшен потенциальный член порядка $\varepsilon^{2}$ (обоснование см. в [25]).

ЗАмЕчАниЕ 5. Поправка на наличие притяжения основывается на аналогичной идее. Мы добавляем в давление член $-\rho r^{2}$ согласно условию (31). К интегрированию по $p$ необходимо добавить интегрирование по $r^{2} d r$, а в экспоненте, аналогично тому, как это сделано выше, добавить член $-p r^{2}+C r^{3}$ и проинтегрировать от нуля до $R$. Появятся два полилогарифма, полученные тем же способом, который описан выше. Главный член будет иметь вид

$$
\begin{aligned}
& -\rho \iint_{0}^{\infty} r^{2} \exp \left\{-\frac{p^{2}}{2 m T_{\mathrm{r}}}-\frac{C r^{3}}{T_{\mathrm{r}}}+\frac{\mu}{T_{\mathrm{r}}}\right\}|p|^{\gamma} r^{2} d|p| d r . \\
& \text { 8. УЧЕТ БЕСКОНЕЧНО МАЛОЙ } \\
& \text { ДИССИПАЦИИ И ПРАВИЛО МАКСВЕЛЛА }
\end{aligned}
$$

\section{8. УЧЕТ БЕСКОНЕЧНО МАЛОЙ ДИССИПАЦИИ И ПРАВИЛО МАКСВЕЛЛА}

В работе [26] рассмотрен переход к двумерному лагранжеву многообразию в четырехмерном фазовом пространстве, где давление $P$ и температура $T$ (интенсивные переменные) играют роль координат, а экстенсивные величины (объем $V$ и энтропия $S$ ) играют роль импульсов от лагранжева многообразия, где энтропия есть порождающее лагранжеву структуру действие. 
Оказывается, это сложное преобразование, приводящее к термодинамическому потенциалу Гиббса, удается провести, как мы видели, лишь с помощью продолжения в область отрицательных энергий. Затем можно обосновать максвелловский переход, введя малую диссипацию (вязкость). Введение бесконечно малой диссипации позволяет одновременно решить проблему критических показателей, не прибегая к гипотезе скейлинга, на которой основан метод ренормгруппы. Покажем это.

В термодинамике вязкость отсутствует. Однако, вообе говоря, без бесконечно малой диссипации равновесие в термодинамике не должно наступить. Поэтому ее введение и затем устремление к нулю естественны.

Геометрическое квантование лагранжева многообразия (см. [27], § 11.4) обычно связывается с введением константы $\hbar$. Винеровским (или туннельным) квантованием автор назвал случай, когда число $ћ$ чисто мнимое [28].

Применим винеровское квантование к термодинамике. Термодинамический потенциал $G=\mu N$ является действием $\mathbb{S}=\int p d q$ на двумерном лагранжевом многообразии $\Lambda^{2}$ в четырехмерном фазовом пространстве $q_{1}, q_{2}, p_{1}, p_{2}$, где $q_{1}$ и $q_{2}-$ соответственно давление $P$ и температура $T$, а $p_{1}$ и $p_{2}$ - объем $V$ и энтропия $S$ с обратным знаком. Все другие потенциалы (внутренняя энергия $E$, свободная энергия $F$ и энтальпия $W$ ) суть результаты проецирования лагранжева многообразия на координатные плоскости $p_{1}, p_{2}$ :

$$
\begin{gathered}
E=-\int \vec{q} d \vec{p}, \quad \vec{q}=\left\{q_{1}, q_{2}\right\}, \quad \vec{p}=\left\{p_{1}, p_{2}\right\} \\
W=-\int\left(q_{2} d p_{2}+q_{1} d p_{1}\right), \quad F=\int\left(q_{1} d p_{1}-q_{2} d p_{2}\right) .
\end{gathered}
$$

При винеровском квантовании имеем

$$
N=\varepsilon \frac{\partial}{\partial \mu}, \quad V=\varepsilon \frac{\partial}{\partial p}, \quad S=-\varepsilon \frac{\partial}{\partial T} .
$$

Следовательно, роль времени $t$ при квантовании играет $\ln (-\mu / T)$ :

$$
G=\mu N \sim \varepsilon \frac{\mu}{T} \frac{\partial}{\partial(\mu / T)}=\varepsilon \frac{\partial}{\partial \ln (\mu / T)} .
$$

Заметим, что туннельное квантование уравнения Ван-дер-Ваальса при $\varepsilon \rightarrow 0$ дает правило Максвелла (см. ниже).

Как мы увидим ниже, критическая точка и точки спинодали являются фокальными, и поэтому при $\varepsilon \rightarrow 0$ они не переходят в "классику" модели Ван-дер-Ваальса. Точки спинодали, подобно точкам поворота в квантовой механике, приближаются функцией Эйри, а критическая точка - точка возникновения двух точек поворота (двух функций Эйри) - приближается функцией Вебера (см. [29]). Именно через функцию Вебера выражается в уравнении Бюргерса точка возникновения ударной волны при $\varepsilon \rightarrow 0$. Если вне этих точек совершить переход при $\varepsilon \rightarrow 0$, то получится модель Ван-дер-Ваальса-Максвелла. Однако в самих этих точках предельный переход нарушается, поэтому так называемые "классические" критические показатели Ландау [28] оказываются грубо не совпадающими с экспериментом. Функция Вебера дает особенности вида $\varepsilon^{-1 / 4}$, а функция Эйри - особенность вида $\varepsilon^{-1 / 6}$. 
Приведем более подробное рассмотрение уравнения Бюргерса.

Рассмотрим уравнение теплопроводности

$$
\frac{\partial u}{\partial t}=\frac{\varepsilon}{2} \frac{\partial^{2} u}{\partial x^{2}}, \quad x \in \mathbb{R}, \quad t \geqslant 0,
$$

где $\varepsilon>0$ - малый параметр. Известно, что все линейные комбинации

$$
u=\lambda_{1} u_{1}+\lambda_{2} u_{2}
$$

решений $u_{1}$ и $u_{2}$ уравнения (49) являются решениями этого уравнения.

Произведем замену $u=e^{-w(x, t) / \varepsilon}$. Получим нелинейное уравнение

$$
\frac{\partial w}{\partial t}+\frac{1}{2}\left(\frac{\partial w}{\partial x}\right)^{2}-\frac{\varepsilon}{2} \frac{\partial^{2} w}{\partial x^{2}}=0,
$$

которое называется проинтегрированным уравнением Бюргерса ${ }^{3)}$. Очевидно, что с любым решением $u_{i}$ уравнения (49) ассоциируется решение $w_{i}=-\varepsilon \ln u_{i}, i=1,2$, уравнения (51). С решением (50) уравнения (49) ассоциируется решение уравнения (51)

$$
w=-\varepsilon \ln \left(e^{-\left(w_{1}+\mu_{1}\right) / \varepsilon}+e^{-\left(w_{2}+\mu_{2}\right) / \varepsilon}\right),
$$

где $\mu_{i}=-\varepsilon \ln \lambda_{i}, i=1,2$. Поскольку

$$
\lim _{\varepsilon \rightarrow 0} w=\min \left(w_{1}, w_{2}\right)
$$

мы получаем $(\min ,+)$ алгебру тропической математики [19].

Для определения решений при $t>t_{\text {c }}$ Хопф предложил рассмотреть уравнение Бюргерса

$$
\frac{\partial v}{\partial t}+v \frac{\partial v}{\partial x}-\frac{\varepsilon}{2} \frac{\partial^{2} v}{\partial x^{2}}=0,\left.\quad v\right|_{t=0}=p_{0}(x),
$$

и назвать (обобщенным) решением уравнения

$$
\frac{\partial p}{\partial t}+p \frac{\partial p}{\partial x}=0,\left.\quad p\right|_{t=0}=p_{0}(x)
$$

функцию $p_{\text {об }}=\lim _{\varepsilon \rightarrow 0} v$ (волны Римана).

Решение $v$ уравнения Бюргерса выражается через логарифмическую производную

$$
v=-\varepsilon \frac{\partial}{\partial x} \ln u
$$

решения $u$ уравнения теплопроводности

$$
\frac{\partial u}{\partial t}=\frac{\varepsilon}{2} \frac{\partial^{2} u}{\partial x^{2}},\left.\quad u\right|_{t=0}=\exp \left\{-\frac{1}{\varepsilon} \int_{-\infty}^{x} p_{0}(x) d x\right\} .
$$

3) Обычное уравнение Бюргерса выводится из уравнения (51) дифференцированием по $x$ и заменой $v=\partial w / \partial x$. 
Таким образом, исходная задача сводится к изучению логарифмического предела решения уравнения теплопроводности. Известно, что решение задачи (56) имеет вид

$$
u=(2 \pi \varepsilon t)^{-1 / 2} \int_{-\infty}^{\infty} \exp \left\{-\left((x-\xi)^{2}+2 t \int_{-\infty}^{\xi} p_{0}(\xi) d \xi\right) / 2 t h\right\} d \xi
$$

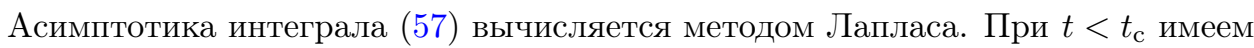

$$
u=\left(|J|^{-1 / 2}(\xi(x, t), t)+O(\varepsilon)\right) e^{-S(x, t) / \varepsilon} .
$$

Здесь $S(x, t)=\int_{-\infty}^{r(t)} p d x$, а интеграл вычисляется вдоль лагранжевой кривой $\Lambda^{t}$, точка $r(x)$ - точка на $\Lambda^{t}$. При $t>t_{\text {с }}$ существуют три точки $r_{1}(x), r_{2}(x), r_{3}(x)$ на $\Lambda^{t}$, проекции которых на ось $x$ совпадают, или, иначе говоря, уравнение $Q(t, \xi)=x$ при $x \in\left(x_{1}, x_{2}\right)$ имеет три решения $\xi_{1}(x, t), \xi_{2}(x, t), \xi_{3}(x, t)$. Обозначим $\widetilde{S}(x, t)=$ $\int_{-\infty}^{r(x)} p d x$ при $x<x_{1}, x>x_{2}$ и $\widetilde{S}(x, t)=\min \left(S_{1}, S_{2}, S_{3}\right), S_{j}=\int_{-\infty}^{r_{j}(x)} p d x$, где $j=1,2,3$ при $x \in\left[x_{1}, x_{2}\right]$.

Приведенные рассуждения позволяют получить обобщенное разрывное решение задачи (54) при временах $t>t_{\mathrm{c}}$. Оно определяется функцией $p=p(x, t)$, задающей существенные области кривой $\Lambda^{t}$ [28]. Заметим, что отсюда, в частности, следует известное в гидродинамике правило равных площадей для определения фронта ударной волны, эволюция которой описывается уравнением (54). Отметим, что это в точности отвечает правилу Максвела для уравнения Ван-дер-Ваальса.

Решение $v=v(x, \varepsilon)$ уравнения Бюргерса в критической точке $x=p^{3}$ вычисляется по формуле

$$
v(x, \varepsilon)=\varepsilon \frac{\partial \ln u(x)}{\partial x}=\int_{0}^{\infty} \exp \left\{\frac{-x \xi-\xi^{4} / 4}{\varepsilon}\right\} \xi d \xi / \int_{0}^{\infty} \exp \left\{\frac{-x \xi-\xi^{4} / 4}{\varepsilon}\right\} d \xi .
$$

При $x \rightarrow 0$ после замены $\xi / \sqrt[4]{\varepsilon}=\eta$ получаем

$$
v(\varepsilon, x) \underset{x \rightarrow 0}{\longrightarrow} \sqrt[4]{\varepsilon} \cdot \text { const. }
$$

Что это означает с точки зрения классики и классического измерения, когда выполнено условие, которое в книге [6] названо "условием квазиклассичности" (это значит, в случае, когда мы находимся вне фокальной точки)? Для преобразования Лапласа это означает, что мы находимся в области, где применим асимптотический метод Лапласа, т. е. где

$$
u(x)=\frac{1}{\sqrt{\varepsilon}} \int_{0}^{\infty} e^{-(p x-\widetilde{S}(p)) / \varepsilon} d p .
$$

Если решение соотношения

$$
x=\frac{\partial \widetilde{S}}{\partial p}
$$

невырожденно, т. е. $\partial^{2} \widetilde{S} / \partial p^{2} \neq 0$ в точке $\partial \widetilde{S} / \partial p=x$, то в этом случае приведенный интеграл (61) при $\varepsilon \rightarrow 0$ ограничен. Чтобы он имел нуль порядка $\varepsilon^{1 / 4}$, мы должны проинтегрировать его по $x$ дробной производной $D^{-1 / 4}$. Значение $D^{-1 / 4}$ применительно к единице дает приблизительно $x^{1 / 4}$. 
В силу приниципа неопределенности [30] соответствие оператора дифференцирования и малого параметра вида $D \rightarrow 1 / \varepsilon$ сохраняется для отношения $-\varepsilon \frac{\partial u / \partial x}{u}$, хотя главный член асимптотики в разности $\frac{\partial^{2} u / \partial x^{2}}{u}$ и $\frac{(\partial u / \partial x)^{2}}{u^{2}}$ сокращается.

ЗАмечАниЕ 6 . Повторим выкладки из работы [6], учитывая, что $\bar{D}$ на этом классе функций имеет свойства

$$
\int \varphi D \varphi d x=\frac{1}{2} \int D \varphi^{2} d x=0, \quad \int x \varphi^{2} d x=0 .
$$

Рассмотрим очевидное неравенство

$$
\int_{-\infty}^{+\infty}\left|a x \psi+\frac{d \psi}{d x}\right|^{2} d x \geqslant 0
$$

где $a$ - произвольная действительная постоянная. При вычислении этого интеграла имеют место следующие соотношения:

$$
\begin{gathered}
\int x^{2}|\psi|^{2} d x=\overline{(\Delta x)^{2}} \\
\int\left(x \frac{d \psi^{*}}{d x} \psi+x \psi^{*} \frac{d \psi}{d x}\right) d x=\int x \frac{d|\psi|^{2}}{d x} d x=-\int|\psi|^{2} d x=-1, \\
\int \frac{d \psi^{*}}{d x} \frac{d \psi}{d x} d x=-\int \psi^{*} \frac{d^{2} \psi}{d x^{2}} d x=\frac{1}{\varepsilon^{2}} \int \psi^{*}|D|^{2} \psi d x=\frac{1}{\varepsilon^{2}} \overline{|\Delta D|^{2}} .
\end{gathered}
$$

Получаем

$$
a^{2} \overline{(\Delta x)^{2}}-a+\frac{1}{\varepsilon^{2}} \overline{|\Delta D|^{2}} \geqslant 0 .
$$

Для того чтобы этот квадратичный (по $a$ ) трехчлен был положительным при любых значениях $a$, должно быть выполнено условие

$$
\overline{4(\Delta x)^{2}} \frac{1}{\varepsilon^{2}} \overline{|\Delta D|^{2}} \geqslant 1
$$

или

$$
\sqrt{\overline{(\Delta x)^{2}} \overline{|\Delta D|^{2}}} \geqslant \frac{\varepsilon}{2}
$$

Таким образом, туннельное квантование объясняет и условие $\mu=0$ для фотонов, и $\mu \leqslant 0$ для бозонов.

В случае термодинамики роль $x$ играет давление $P$, а роль импульса $p$ играет объем $V$. Поэтому $V \sim P^{1 / 4}$, т. е.

$$
P_{\mathrm{c}} \sim\left(V-V_{\mathrm{c}}\right)^{4} .
$$

Это и есть скачок критического показателя. Аналогично можно получить и другие критические показатели [30] (о сравнении с экспериментальными данными см. ту же работу). 


\section{9. БОЛЕЕ ГРУБЫЙ ПРИБОР И ВЫВОД ЗАКОНА СООТВЕТСТВЕННЫХ СОСТОЯНИЙ}

В свое время атомных микроскопов не было, и приборы не могли различать частицы воздуха и видеть в нем углекислый газ и другие элементы. С их помощью экспериментаторы считали плотность воздуха, полагая его идеальным газом. Фазовый переход воздуха в жидкость стали наблюдать, когда научились различать разные молекулы воздуха. Но если использовать старый прибор, который не видит различий между элементами воздуха или смеси газов с одинаковой концентрацией, то фазовый переход будет более "размазанный".

Мы сравнивали винеровское квантование термодинамики с диссипацией, приводящей к ударной волне. Но диссипация у разных веществ может быть разная, а нас интересуют условия Гюгонио для всей смеси и мы не хотим различать стратификационно возникающие внутренние ударные волны внутри общей ударной волны. Это особенно ярко проявляется в случае, когда диссипативные параметры вязкости и теплопроводности разные (см. [7], § 95): вязкость $\nu$ мала, а теплопроводность $\chi \gg \nu$. Если процессы внутри ударной волны нас не интересуют, то мы ставим условия Гюгонио на концах ударной волны, "размазанной" из-за высокого значения теплопроводности.

В качестве грубого примера можно взять отвес, гирьку на шнурке, которые используются при строительстве в качестве прямой, перпендикулярной поверхности земли. Более точное измерение учитывает колебания воздуха и структуру шнурка. Точно так же в более грубой термодинамике, не различающей молекулы (см. рис. 10), принцип неопределенности (66) дает сильно "размазанные" изотермы, поскольку при рассмотрении задачи с более грубым прибором мы уже не имеем права устремлять диссипацию к нулю.

В наборе газов мы должны выбрать эталонный газ, в котором разница, например, с точки зрения количества димеров между паром и жидкостью наименьшая. Таким газом являются пары ртути $\left(Z_{\mathrm{c}}=0.4\right)$. Для изотермы этого газа мы произведем нормировку активности (см. равенства (24), (25) при $Z=0.4$ и будем полагать, что фазового перехода на критической изотерме ртути нет. Тогда все остальные газы в их совокупности при учете (66) будут испытывать на критических изотермах фазовый переход ${ }^{4)}$ в “жидкость". Прибор не различает данный набор молекул, и мы вычисляем среднюю степень свободы, взяв арифметическое среднее степеней свободы молекул, которые мы вычисляем исходя из экспериментальных данных для $Z_{\text {c }}$ рассматриваемых газов. Наибольшее значение $Z_{\text {c }}=0.4$ у ртути $(\mathrm{Hg})$, поэтому среднее число степеней свободы этого набора молекул заведомо меньше, следовательно, $Z_{\text {average }}<Z_{\text {c }}$ (для ртути), $\gamma<\gamma_{\mathrm{Hg}}$. Критическое давление больше, чем у ртути: $\zeta\left(\gamma_{\mathrm{Hg}}+2\right)<\zeta\left(\gamma_{\text {average }}+2\right)$. Следовательно, выбранное нами по ртути значение $P_{\mathrm{r}}=1$ меньше, чем $P_{\text {average }}=\zeta\left(\gamma_{\text {average }}+2\right) / \zeta\left(\gamma_{\mathrm{Hg}}+2\right)$. Значит, значение $P_{\mathrm{r}}=1$ для $Z_{\mathrm{Hg}}$ лежит в области фазового перехода "газ-жидкость" для $Z_{\text {average }}$. Из этого следует, что при $P_{\mathrm{r}}=1$ происходит фазовый переход в жидкость у среднего значения $Z_{\text {average }}$ Бозе-конденсат, разумеется, возникает также в случае, когда плотность считается по всем частицам, неразличимым нашим прибором.

\footnotetext{
4) Разрыв производной незаметен из-за условия (66).
} 
Этот фазовый переход в жидкость виден на рис. 10, где жидкость изображена жирной наклонной линией ${ }^{5)}$.

Перейдем к выводу закона соответственных состояний, в котором участвуют все газы, которые прибор не различает.

Заметим, во-первых, что теория чисел для дробной размерности дает распределение, где не участвует объем $V$, так же как и исходные соотношения в [7] и для идеального газа Больцмана-Максвелла и для идеального газа Бозе-Эйнштейна. Далее в работе [7] распределение как для бозе-частиц, так и для фотонов умножается на трехмерный объем $V$. Основным распределением, разумеется, является распределение без объема $V$, и умножение его на $V$ диктуется соответствием с термодинамикой, в которой пара объем-давление является важнейшим инструментом.

Поэтому наиболее естественным обобщением на неидеальное распределение является умножение дробного бозе-распределения на функцию от $V$ вида $V \varphi\left(V / V_{0}\right)$, где $V_{0}$ - некоторый эталонный объем, а функция $\varphi(x)$ гладкая.

Тогда потенциал $\Omega$ для дробной размерности принимает вид

$$
\begin{aligned}
\Omega(\mu, T)= & -\Lambda^{\gamma+1} V \varphi\left(V / V_{0}\right) \frac{T^{\gamma+2}}{\Gamma(\gamma+2)} \int_{0}^{\infty} \frac{t^{\gamma+1} d t}{\left(e^{t} / y\right)-1}= \\
& =-\Lambda^{\gamma+1} T^{\gamma+2} V \varphi\left(V / V_{0}\right) \operatorname{Li}_{\gamma+2}(y),
\end{aligned}
$$

где $y=e^{\mu / T}-$ активность, $\mu-$ химический потенциал.

Выпишем соотношение на Zeno line:

$$
\begin{gathered}
T_{\mathrm{z}}=T_{\mathrm{B}}\left(1-\frac{\rho_{\mathrm{z}}}{\rho_{\mathrm{B}}}\right), \\
P_{\mathrm{z}}=\rho_{\mathrm{z}} T_{\mathrm{B}}\left(1-\frac{\rho_{\mathrm{z}}}{\rho_{\mathrm{B}}}\right),
\end{gathered}
$$

где индексом z мы помечаем, что эти значения берутся на Zeno line, т. е. при $Z=1$.

Построим соотношение $Z=1$ на Zeno line. Оно будет иметь вид

$$
Z=\frac{\partial \Omega / \partial V}{T_{\mathrm{z}} \partial \Omega / \partial \mu}=\frac{V_{\mathrm{z}}\left[\varphi\left(V_{\mathrm{z}} / V_{0}\right)+\left(V_{\mathrm{z}} / V_{0}\right) \varphi^{\prime}\left(V_{\mathrm{z}} / V_{0}\right)\right]}{V_{\mathrm{z}} \varphi\left(V_{\mathrm{z}} / V_{0}\right)} \frac{\mathrm{Li}_{\gamma+2}\left(y_{\mathrm{z}}\right)}{\mathrm{Li}_{\gamma+1}\left(y_{\mathrm{z}}\right)}=1 .
$$

Из (70) на Zeno line получаем

$$
T_{\mathrm{z}}^{2+\gamma}\left[\varphi\left(\frac{V_{\mathrm{z}}}{V_{0}}\right)+\left(\frac{V_{\mathrm{z}}}{V_{0}}\right) \varphi^{\prime}\left(\frac{V_{\mathrm{z}}}{V_{0}}\right)\right] \operatorname{Li}_{\gamma+2}\left(y_{\mathrm{z}}\right)=\left(\frac{N}{V_{\mathrm{z}}}\right) T_{\mathrm{B}}\left(1-\frac{N}{V_{\mathrm{z}} \rho_{\mathrm{B}}}\right) .
$$

Из (69) получаем

$$
T_{\mathrm{B}}^{\gamma+1}\left(1-\frac{N}{V_{\mathrm{z}} \rho_{\mathrm{B}}}\right)^{\gamma+1} \varphi\left(\frac{V_{\mathrm{z}}}{V_{0}}\right) \operatorname{Li}_{\gamma+1}\left(y_{\mathrm{z}}\right)=\frac{N}{V_{\mathrm{z}} \rho_{\mathrm{B}}} .
$$

\footnotetext{
5) Строго доказать существование фазового перехода можно лишь для нового идеального газа в

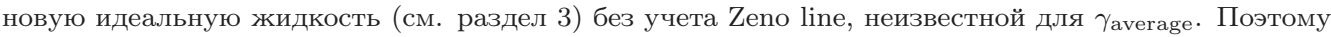
строго доказанный переход от $Z=0.4, P_{\mathrm{r}}=1$ в жидкость будет получаться несколько выше, чем $P_{\mathrm{r}}=1, Z=0.12$ на рис. 10 .
} 
Здесь $N / V_{\mathrm{z}}=\mathrm{const}, N=\mathrm{const}$, и, следовательно, это уравнение содержит неизвестные $y_{\mathrm{z}}, V_{z}$ и $\varphi\left(V_{\mathrm{z}} / V_{0}\right)$.

Найдя значение $y_{\mathrm{z}}$ как функцию от $\varphi\left(V_{\mathrm{z}} / V_{0}\right)$ и $V_{z}$, мы подставляем его в формулу (71), превратив тем самым уравнение (71) в дифференциальное уравнение относительно функции $\varphi(x)$, зависящее от констант $\rho_{\mathrm{B}}, T_{\mathrm{B}}$. При каких условиях решение этого уравнения существует?

Введем обозначения: $T_{\mathrm{s}}=T_{\text {standard }}=T / T_{\mathrm{m}}, P_{\mathrm{s}}=P_{\text {standard }}=P / P_{\mathrm{m}}$, где $T_{\mathrm{m}}$ и $P_{\mathrm{m}}$ определяются из формул

$$
Z_{\mathrm{m}}=\frac{P_{\mathrm{m}}}{\rho_{\mathrm{m}} T_{\mathrm{m}}}, \quad P_{\mathrm{m}}=T_{\mathrm{m}}^{\gamma+2}, \quad V_{0}=V_{\mathrm{m}}=V_{\mathrm{max}},
$$

a $Z_{\mathrm{m}}=Z_{\max }$ и $\rho_{\mathrm{m}}=\rho_{\min }$ определены ниже. В силу того, что $N_{\mathrm{c}}$ не зависит от $T$

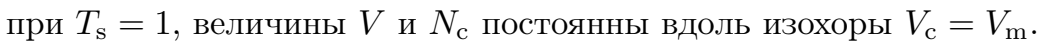

Выпишем эти соотношения в точке $T_{\mathrm{s}}=1, \quad P_{\mathrm{s}}=1$ :

$$
\begin{gathered}
{\left[\varphi\left(\frac{V_{\mathrm{z}}}{V_{0}}\right)+\left(\frac{V_{\mathrm{z}}}{V_{0}}\right) \varphi^{\prime}\left(\frac{V_{\mathrm{z}}}{V_{0}}\right)\right] \zeta(\gamma+2)=1, \quad V_{\mathrm{m}}=V_{\max }} \\
V_{\mathrm{m}} \varphi(1) \zeta(\gamma+1)=N_{\mathrm{c}} .
\end{gathered}
$$

Следовательно,

$$
Z_{\mathrm{m}}=\frac{V_{\mathrm{m}}}{N} \frac{\zeta(\gamma+1)}{\zeta(\gamma+2)} \frac{\operatorname{Li}_{\gamma+2}(1)}{\mathrm{Li}_{\gamma+1}(1)}=\frac{V_{\mathrm{m}}}{N_{\mathrm{c}}}=\frac{1}{\rho_{\mathrm{m}}} .
$$

Поскольку мы строим изохоры $V=$ const в плоскости $\{Z, P\}$, то $V_{\mathrm{m}}=V_{\mathrm{z}}$, и, исключая $\left(V_{\mathrm{z}} \varphi\left(V_{\mathrm{z}} / V_{0}\right)^{\prime}\right.$ из $(73)$ в силу $(75)$, получаем

$$
T_{\mathrm{B}}^{\gamma+1}\left(1-\frac{1}{Z_{\mathrm{m}} \rho_{\mathrm{B}}}\right)^{\gamma+1} \frac{1}{\zeta(\gamma+2)} \operatorname{Li}_{\gamma+2}\left(y_{\mathrm{z}}\right)=\frac{1}{Z_{\mathrm{m}}} .
$$

Поскольку $V_{\mathrm{m}}=V_{\mathrm{z}}$, то и

$$
\varphi\left(\frac{V_{\mathrm{z}}}{V_{\mathrm{m}}}\right)=\varphi(1)=\frac{N_{\mathrm{c}}}{\zeta(\gamma+1) V_{\mathrm{m}}},
$$

a

$$
\varphi(1)+\varphi^{\prime}(1)=\frac{1}{\zeta(\gamma+2)},
$$

и из (71) следует соотношение на Zeno line

$$
\frac{\operatorname{Li}_{\gamma+2}\left(y_{\mathrm{z}}\right)}{\operatorname{Li}_{\gamma+1}\left(y_{\mathrm{z}}\right)} \frac{V_{\mathrm{m}}}{N_{\mathrm{c}}} \frac{\zeta(\gamma+1)}{\zeta(\gamma+2)}=1
$$

Исключая $y_{\mathrm{z}}$, мы получаем соотношение для $Z_{\mathrm{m}}$ и $\gamma$. Максимальное значение $Z_{\mathrm{m}}$ зависит только от $\rho_{\mathrm{B}}$ и $T_{\mathrm{B}}$. Для ртути оно получается при $\gamma_{m}=\gamma_{\min }=0.1$, а

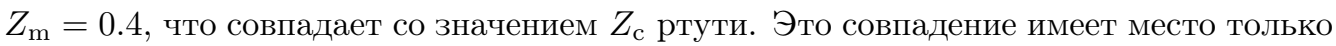
для ртути $\mathrm{Hg}$ из всех элементов таблицы Менделеева, что подтверждает верный выбор потенциала $\Omega(68)$. 


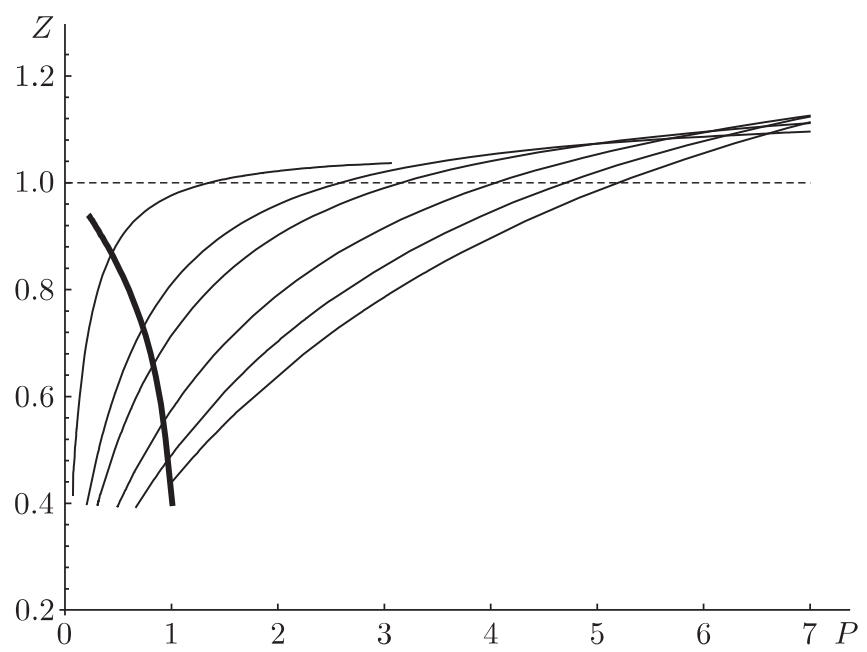

Рис. 12. Штриховая прямая - Zeno line $Z=1$. Жирная линия - критическая изотерма реального газа (ртути $\mathrm{Hg}$ ), вычисленная теоретически; тонкие линии - изохоры $\mathrm{Hg}$ при $T<T_{\mathrm{c}}$. Их начальные точки лежат на газовой спинодали.

Мы получаем, что при $T_{\mathrm{s}}=1$ и $P_{\mathrm{s}}=1$ максимальное значение $Z_{\mathrm{m}}$ (а значит, и максимальный удельный объем для дифференциальных соотношений (71)-(73)) для критической изотермы $Z_{\mathrm{c}}=0.4 \mathrm{c} T_{\mathrm{s}}=1, P_{\mathrm{s}}=1$ получается при малом $\gamma$. Таким образом, уравнения (71)-(73) справедливы лишь при $V / N>V_{\mathrm{m}} / N_{\mathrm{c}}$. Тогда в качестве начального условия для функции $\varphi(1)$ мы можем брать (78) при $V / N=Z_{\mathrm{m}}$. Семейство изохор согласно системе (72)-(73) с указанным начальным условием изображено на рис. 12.

Для предельной изохоры $V / N=Z_{\mathrm{m}}$ уравнение при $\gamma=\gamma_{\mathrm{m}}$ имеет вид

$$
\begin{gathered}
Z=Z_{\mathrm{m}} \frac{\zeta(\gamma+1)}{\zeta(\gamma+2)} \frac{\operatorname{Li}_{\gamma+2}(y)}{\operatorname{Li}_{\gamma+1}(y)}, \quad 1 \leqslant y \leqslant y_{\mathrm{z}}(\gamma), \\
P_{\mathrm{s}}=T_{\mathrm{s}}^{\gamma+2} \operatorname{Li}_{\gamma+2}(y), \\
N_{\mathrm{c}}=T_{\mathrm{s}}^{\gamma+1} \operatorname{Li}_{\gamma+1}(y) V_{\mathrm{m}} .
\end{gathered}
$$

Следовательно,

$$
P_{\mathrm{s}}=\left(\frac{1}{Z_{\mathrm{m}} \operatorname{Li}_{\gamma+1}(y)}\right)^{(\gamma+2) /(\gamma+1)} \frac{\operatorname{Li}_{\gamma+2}(y)}{\zeta(\gamma+2)}, \quad 1 \leqslant y \leqslant y_{\mathrm{z}}(\gamma)
$$

Уравнения (81) и (82) дают почти прямой отрезок изохоры.

Начиная от $Z<0.4$ (например, для газа Ван-дер-Ваальса) происходит фазовый переход в жидкость нового типа для неразличимых частиц закона соответственных состояний. Он дает широкую (за счет принципа неопределенности и достаточно большой диссипации) область вокруг отрезка прямой $P_{\mathrm{s}}=1, T_{\mathrm{s}}=1, Z<0.4$. 


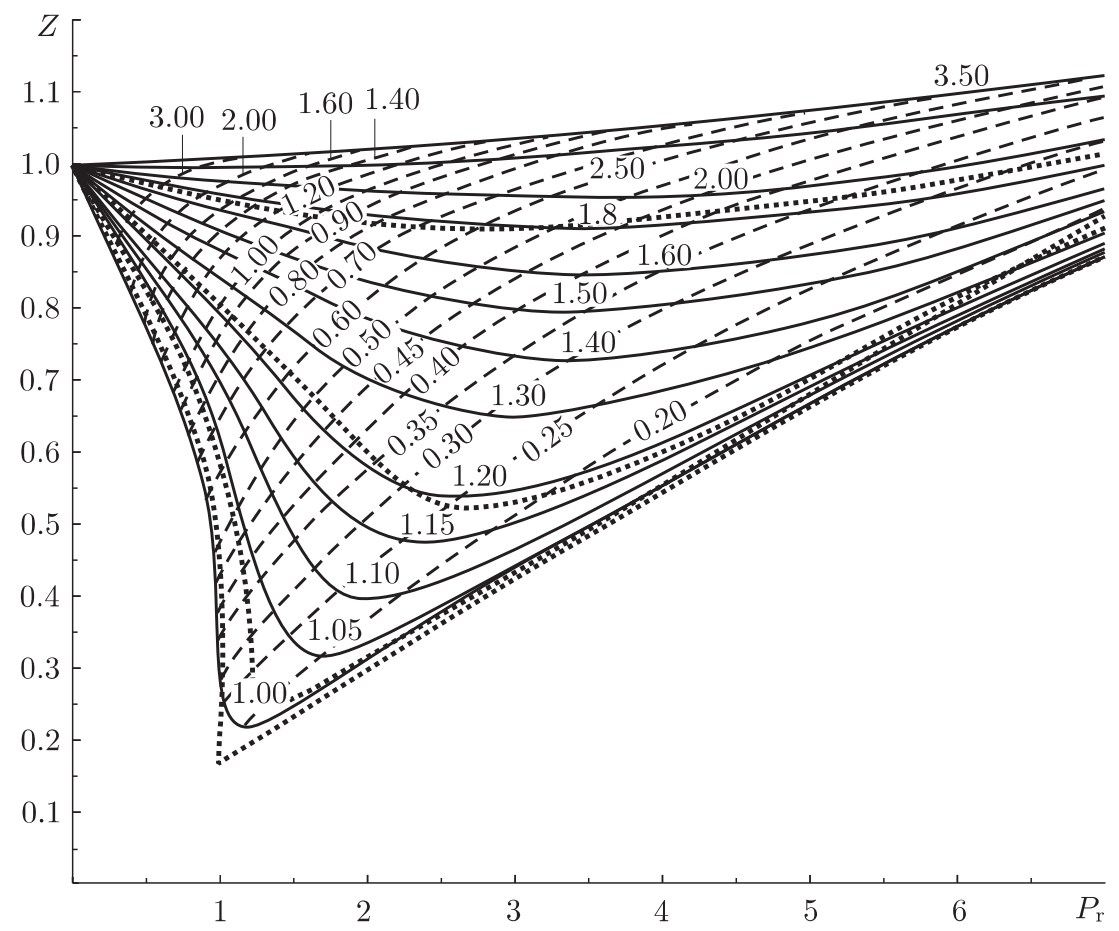

Рис. 13. Сплошные линии - экспериментальные изотермы для метана, штриховые линии построены на основе теоретических данных. Числа у штриховых кривых - значения $V / R$.

ЗАмЕчАниЕ 7. Поскольку самая крайняя изохора (на рис. 12 она не изображена) является отрезком прямой, то все изохоры большой плотности также должны быть отрезками прямых. Они проходят через точку $\rho>\rho_{\mathrm{m}}$ на Zeno line и точку $Z_{0}=1 / \rho$ на прямой $P_{\mathrm{s}}=1$. Мы получаем, таким образом, включая изохоры, изображенные на рис. 12 , полное семейство изохор при $Z \leqslant 1, P_{\mathrm{s}} \geqslant 1$, отвечающих закону соответственных состояний. Каждой точке изохоры в плоскости $\{Z, P\}$ отвечает точка температуры, и мы строим изотермы, которые с точностью до винеровского принципа неопределенности соответствуют экспериментальному закону соответственных состояний (рис. 13).

Как мы уже отмечали, винеровское квантование термодинамики сглаживает острые углы на критических изотермах закона соответственных состояний аналогично тому, как наличие вязкости сглаживает ударную волну.

Экспериментаторы, проводя эксперимент, не могут, как правило, различать квантовые эффекты и эффекты вязкости в общей картине закона соответственных состояний. Но принцип неопределенности Виннера сказывается на эксперименте и способствует тому, что для разных газов мы получаем схожую картину.

Благодарности. Автор выражает глубокую благодарность В. С. Воробьеву за постоянные дискуссии, проверку всех вычислений и построение графиков. Работа выполнена при поддержке РФФИ (грант № 11-01-12058-офи_м). 


\section{Список литературы}

[1] В. П. Маслов, С. Э. Таривердиев, Итоги науки и техн. Сер. Теор. вероятн. Матем. стат. Теор. кибернет., 19, ВИНИТИ, М., 1982, 85-125.

[2] В. П. Маслов, О. Ю. Шведов, Метод комплексного ростка в задаче многих частии, и в квантовой теории поля, Эдиториал УРСС, М., 2000.

[3] В. П. Маслов, Комплексный метод ВКБ в нелинейных уравнениях, Наука, М., 1977.

[4] V.P. Maslov, Russ. J. Math. Phys., 15:4 (2008), 493-510.

[5] В. П. Маслов, Т. В. Маслова, ТВП, 57:3 (2012), 471-498.

[6] Л. Д. Ландау, Е. М. Лифшиц, Теоретическая физика, т. 3: Квантовая механика. Нерелятивистская теория, Наука, М., 1974.

[7] Л. Д. Ландау, Е. М. Лифшиц, Статистическая физика, Наука, М., 1964.

[8] А.Н. Ширяев, Вероятность, т. 1: Элементарная теория вероятностей. Математические основания. Предельные теоремы, МЦНМО, М., 2004.

[9] P. Erdős, J. Lehner, Duke Math. J., 8:2 (1941), 335-345.

[10] V.P. Maslov, Math. Notes, 91:5-6 (2012), 603-609.

[11] Н. Н. Боголюбов, "Квазисредние в задачах статистической механики”, Избранные труды в трех томах, т. 3, Наукова думка, Киев, 1971, 174-243.

[12] В. Я. Френкель, Яков Ильич Френкель, Наука, М.-Л., 1966.

[13] E. M. Apfel'baum, V.S. Vorob'ev, Russ. J. Math. Phys., 18:1 (2011), 26-32.

[14] V.P. Maslov, Threshold levels in economics, arXiv: 0903.4783.

[15] К. И. Шмулович, Л. Меркьюри, Вестник Отделения наук о Земле РАН, 1:24 (2006), $1-3$.

[16] V.P. Maslov, P.P. Mosolov, Nonlinear Wave Equations Perturbed by Viscous Term, De Gruyter Expositions in Mathematics, 31, Walter de Gruyter, Berlin, 2000.

[17] S. T. Kuroda, Scattering theory for differential operators. III, Lecture Notes in Mathematics, 448, Springer, Berlin.

[18] G. A. Martynov, Fundamental Theory of Liquids: Method of Distribution Functions, Adam Hilger, Bristol, 1992.

[19] Г. Л. Литвинов, Зап. научн. сем. ПОМИ, 326 (2005), 145-182.

[20] E. M. Apfelbaum, V.S. Vorob'ev, J. Phys. Chem. B, 113:11 (2009), 3521-3526.

[21] В. П. Маслов, ТМФ, 161:3 (2009), 420-458.

[22] E. M. Apfelbaum, V.S. Vorob'ev, G. A. Martynov, J. Phys. Chem. B, 110:16 (2006), 8474-8480.

[23] В. П. Маслов, Матем. заметки, 83:3 (2008), 465-467.

[24] V.P. Maslov, Russ. J. Math. Phys., 18:4 (2011), 440-464.

[25] V.P. Maslov, Math. Notes, 92:5-6 (2012), 657-663.

[26] V.P. Maslov, A.S. Mischenko, Russ. J. Math. Phys., 10:2 (2003), 161-172.

[27] Н. Харт, Геометрическое квантование в действии, Мир, М., 1985.

[28] В. П. Маслов, Асимптотические методы и теория возмущений, Наука, М., 1988.

[29] В. П. Маслов, УМН, 38:6(234) (1983), 3-36.

[30] В. П. Маслов, ТМФ, 170:3 (2012), 457-467. 